\title{
SINGULARITIES OF STATIONARY SOLUTIONS TO THE VLASOV-POISSON SYSTEM IN A POLYGON
}

\author{
FAHD KARAMI \\ Ecole Supérieure de Technologie d'Essaouira, Université Cadi Ayyad \\ B.P 383 Essaouira El Jadida, Essaouira, Morocco \\ fa.karami@ucam.ac.ma \\ SIMON LABRUNIE \\ Institut Élie Cartan (Mathématiques) UMR 7502, Université de Lorraine, CNRS \\ 8 INRIA (project-team CALVI) \\ 54506 Vandouvre-lès-Nancy cedex, France \\ Simon.Labrunie@univ-lorraine.fr \\ BRUNO PINÇON \\ Institut Élie Cartan (Mathématiques) UMR 7502, Université de Lorraine, CNRS \\ E) INRIA (project-team CORIDA) \\ 54506 Vandouvre-lès-Nancy cedex, France \\ Bruno.Pincon@univ-lorraine.fr \\ Received (Day Month Year) \\ Revised (Day Month Year) \\ Communicated by (xxxxxxxxx)
}

\begin{abstract}
We present an existence result for the stationary Vlasov-Poisson system in a bounded domain of $\mathbb{R}^{N}$, with more general hypotheses than considered so far in the literature. In particular, we prove the equivablalence of the kinetic approach (which consists in looking for the equilibrium distribution function) and the potential approach (where the unknown is the electrostatic potential at equilibrium). We study the dependence of the solution on parameters such as the total mass of the distribution, or those entering in the boundary conditions of the potential. Focusing on the case of a plane polygon, we study the singular behavior of the solution near the reentrant corners, and examine the dependence of the singularity coefficients on the parameters of the problem. Numerical experiments illustrate and confirm the analysis.
\end{abstract}

Keywords: Vlasov-Poisson system, Stationary solutions, Corner singularity, Nonlinear elliptic problem, Large solutions.

AMS Subject Classification: 35B40, 35B65, 35J91, 82B40, 82D10, 90C25

\section{Introduction.}

The interplay between electrically charged gases and strong electromagnetic fields is a complex physical issue. The fields further ionization, which in turn modifies the dielectric properties and conductivity of the gas, leading to a non-linear feedback 
on the fields. Among the mechanisms capable of generating strong fields in the first place, the point effect is particularly efficient. This is the principle of the lightning conductor, and is also used in various analogue electronic or microwave devices. The mathematical modelling of such devices requires to investigate the effects of geometrical singularities (edges and vertices on the boundary of the domain of interest) on the solution to kinetic equations, e.g., the Vlasov-Poisson or VlasovMaxwell systems. Singularities are well-described for most linear problems; they can be considered as completely known for the Poisson equation with usual boundary conditions. ${ }^{11,12}$ On the other hand, taking account of boundary conditions in kinetic models is generally quite technical.

Another difficulty, especially from a computational point of view, is that the characteristic time scales associated to the various particle species in the gas are typically several order of magnitude apart. ${ }^{6,17}$ Electrons are very much lighter, and thus faster, than ions (and neutral atoms if any). However, this fact allows one to simplify the model when the time scale of the phenomenon under study is taken into account. For instance, if the relevant time scale is much smaller than that of ion motion, the latter can be neglected, or at least considered as a data, on which the electrons have no effect. On the other hand, on a time scale much larger than that of the electrons, one can consider them to be at any time in thermodynamic equilibrium. ${ }^{6}$ Thus, their distribution function $f$ depends on the one-particle energy only: typical cases are the Maxwell-Boltzmann distribution $f(W) \propto \mathrm{e}^{-W / \theta}$ (where $\theta$ represents the temperature), or the quantum Fermi-Dirac distribution.

In this article, we shall investigate the effect of singularities on a simple model which incorporates both approximations above. In other words, we look at the gas on a time scale much larger than that of electrons, but much smaller than that of ions. One thus obtains a static model, which describes the equilibrium of the electrons in the potential created by both species of particles, while the ion density is given. The treatment of boundary conditions is much easier than in a full kinetic model, but the non-linear character is kept. This may be a first step toward a quasiequilibrium model ${ }^{\mathrm{a}}$ valid on a larger time scale, where the equilibrium description of the electrons would be coupled to a kinetic or fluid model for the ions. This approximation is classical in plasma physics. ${ }^{6}$

The article is written as follows. Section 2 is devoted to the derivation of the static model as a class of stationary solutions to the Vlasov-Poisson system. Its wellposedness is proved in Section 3. This part is strongly reminiscent of Refs. 8, 5, 2, but our assumptions are more general, and we expose certain details and subtleties which were overlooked in the above references. Furthermore, we demonstrate the equivalence of the kinetic approach (which consists in looking for the equilibrium distribution function as the minimum of a free energy functional) and the potential

aSuch models are sometimes called quasi-neutral; we avoid this word as it is also often used in a different and incompatible sense: see Ref. 17 for a numerical study of this issue. 
approach (where the unknown is the electrostatic potential at equilibrium). As the latter is solution to an elliptic equation, it is possible to use the powerful elliptic theory. As a first application, we study the dependence of the solution on parameters such as the total mass of the distribution, or the boundary condition of the potential.

The rest of the article is devoted to the case where the problem is set in a polygonal domain of $\mathbb{R}^{2}$. Section 4 describes the singular behavior of the solution near a non-convex geometrical singularity (i.e., a reentrant corner). The case of the Maxwell-Boltzmann distribution is examined in Section 5, where we study the dependence of the singularity coefficients on the parameters of the problem. The analysis makes use of the theory of large solutions to nonlinear elliptic problems, ${ }^{18,9}$ which we adapt to the case of a polygon. To illustrate the theory, numerical experiments have been conducted, using the Singular Complement Method, ${ }^{7}$ which is particularly well-suited to the problem. The results are exposed in Section 6 .

\section{Stationary solutions to the Vlasov-Poisson system}

\subsection{Setting of the problem}

We consider a population of charged particles, with charge $q$ and mass $m$, described as usual by their distribution function $f(t, \boldsymbol{x}, \boldsymbol{v})$, where $t, \boldsymbol{x}$ and $\boldsymbol{v}$ are the time, position and velocity variables. The particles occupy a domain $\Omega \subset \mathbb{R}^{N}$; they are assumed to be non-relativistic and move in an electrostatic field $\boldsymbol{E}=-\nabla_{x} V(t, \boldsymbol{x})$. The distribution function is governed by the Vlasov equation:

$$
\frac{\partial f}{\partial t}+\boldsymbol{v} \cdot \nabla_{x} f-\frac{q}{m} \nabla_{x} V \cdot \nabla_{v} f=0, \quad \text { in }(0, T) \times \Omega \times \mathbb{R}^{N} .
$$

The potential $V$ is created by the particles under consideration, and possibly by a neutralising background of particles of the opposite sign, ${ }^{\mathrm{b}}$ with given density $n_{\mathrm{e}}(t, \boldsymbol{x})$, and/or an applied voltage $V_{\text {in }}(t, \boldsymbol{x})$ imposed on part of the boundary of $\Omega$. Elsewhere, the boundary conditions are assumed to be homogeneous Dirichlet or Neumann. Summarising, the potential obeys at any time the Poisson equation:

$$
\begin{aligned}
& \Delta_{x} V=\frac{q}{\varepsilon_{0}}\left(n_{\mathrm{e}}-\int f \mathrm{~d} \boldsymbol{v}\right) \text { in }(0, T) \times \Omega ; \\
& V=0 \text { on }(0, T) \times \Gamma_{1}, \quad V=V_{\text {in }} \text { on }(0, T) \times \Gamma_{2}, \quad \partial_{\nu} V=0 \text { on }(0, T) \times \Gamma_{3} .
\end{aligned}
$$

Above, we have of course $\partial \Omega:=\Gamma_{1} \cup \Gamma_{2} \cup \Gamma_{3} ; \varepsilon_{0}$ is the dielectric permittivity of vacuum. We use the shorthand $\int f \mathrm{~d} \boldsymbol{v}$ to denote the mapping $(t, \boldsymbol{x}) \mapsto \int_{\mathbb{R}^{N}} f(t, \boldsymbol{x}, \boldsymbol{v}) \mathrm{d} \boldsymbol{v}$; this function is called the spatial density of particles.

The Vlasov-Poisson system (2.1)-(2.3) must be supplemented with an initial condition

$$
f(0, \boldsymbol{x}, \boldsymbol{v})=f_{0}(\boldsymbol{x}, \boldsymbol{v}) \quad \text { in } \Omega \times \mathbb{R}^{N}
$$

${ }^{\mathrm{b}}$ Equation (2.2) assumes that the charge of these particles is exactly $-q$. Otherwise, one arrives at the same expression after suitably rescaling the data $n_{\mathrm{e}}$. 
as well as suitable boundary conditions for $f$ on $\partial \Omega$, and decay conditions at infinity for $V$ if $\Omega$ is unbounded. We denote by $\mathcal{M}$ the total number of particles, which is preserved by time evolution, at least for not too weak solutions:

$$
\forall t \geq 0, \quad \int_{\Omega} \int_{\mathbb{R}^{N}} f(t, \boldsymbol{x}, \boldsymbol{v}) \mathrm{d} \boldsymbol{v} \mathrm{d} \boldsymbol{x}=\int_{\Omega} \int_{\mathbb{R}^{N}} f_{0}(\boldsymbol{x}, \boldsymbol{v}) \mathrm{d} \boldsymbol{v} \mathrm{d} \boldsymbol{x}:=\mathcal{M} .
$$

We shall also refer to $\mathcal{M}$ as the "mass", as it is proportional to the total mass of the particles. Generally speaking, the existence and uniqueness of solutions is a difficult problem when $\Omega$ is not the whole space $\mathbb{R}^{N}$. Let us mention the works by Guo ${ }^{13,14}$ and Hwang ${ }^{15,16}$ dealing respectively with the cases of a half-space and a smooth convex domain. In both cases, rather restrictive conditions are imposed on the initial data $f_{0}$. Existence (but not uniqueness) of weaker solutions has been proved by Bostan, ${ }^{4}$ but the smoothness of $\Omega$ remains a crucial assumption.

In this article, we are interested in stationary solutions (or equilibria) to (2.1)(2.3), i.e., those which do not depend on time $t$. Obviously, the existence of such solutions supposes that $n_{\mathrm{e}}$ and $V_{\text {in }}$ are independent of $t$. In this case, the solution $V$ to (2.2)-(2.3) is time-independent, and any function of the one-particle energy, namely, $f(\boldsymbol{x}, \boldsymbol{v})=\mathcal{F}\left(\frac{1}{2} m|\boldsymbol{v}|^{2}+q V(\boldsymbol{x})\right)$ is a stationary solution to (2.1). We choose to write this function as:

$$
\mathcal{F}(W):=\gamma(W / \theta-\beta),
$$

where: $\gamma(\cdot)$ is a given function which defines the type of equilibrium; $\theta$ is a "temperature" parameter or typical value of the energy, also supposed to be given; $\beta$ is an unknown scalar, to be determined by the condition:

$$
\int_{\Omega} \int_{\mathbb{R}^{N}} f(\boldsymbol{x}, \boldsymbol{v}) \mathrm{d} \boldsymbol{v} \mathrm{d} \boldsymbol{x}=\mathcal{M} .
$$

In the stationary framework, the total mass is not inherited from the initial condition, but has to be supplied as a data of the problem, i.e., the integral of the solution to (2.1) must match the actual number of particles under consideration.

The existence and stability of stationary solutions have been examined ${ }^{8,5}$ when $\Omega=\mathbb{R}^{N}$, and when $\Omega$ is bounded. ${ }^{2}$ In this article, we shall give a more general version of the existence and uniqueness proof in a bounded domain.

\subsection{Rescaled equations and basic notations}

To simplify the notations while keeping track of the physical parameters, we now derive a rescaled version of the equations. To this end, we introduce some units of length $\bar{x}$ and density $\bar{n}$. The temperature parameter $\theta$ introduced in (2.4) furnishes the units of speed and potential:

$$
\bar{v}=\sqrt{\frac{\theta}{m}}, \quad \bar{V}=\frac{\theta}{|q|} ;
$$

the unit of distribution function is $\bar{f}=\bar{n} / \bar{v}^{N}$. Furthermore, let $\delta$ be the sign of $q$. 
Keeping the same notation for the rescaled variables and domain, we arrive at the following equations:

$$
\begin{aligned}
& f(\boldsymbol{x}, \boldsymbol{v})=\gamma\left(\frac{1}{2}|\boldsymbol{v}|^{2}+\delta V(\boldsymbol{x})-\beta\right), \quad \forall(\boldsymbol{x}, \boldsymbol{v}) \in \Omega \times \mathbb{R}^{N} ; \\
& \Delta_{x}(\delta V)=\eta\left(n_{\mathrm{e}}-\int f \mathrm{~d} \boldsymbol{v}\right) \text { in } \Omega, \quad \text { where: } \eta:=q^{2} \bar{n} \bar{x}^{2} /\left(\varepsilon_{0} \theta\right) ; \\
& \int_{\Omega} \int_{\mathbb{R}^{N}} f(\boldsymbol{x}, \boldsymbol{v}) \mathrm{d} \boldsymbol{v} \mathrm{d} \boldsymbol{x}=M:=\frac{\mathcal{M}}{\bar{n} \bar{x}^{N}}
\end{aligned}
$$

the boundary conditions (2.3) being still valid in rescaled units. If the dimension $N$ is different from 2 , it is possible to choose the units $(\bar{n}, \bar{x})$ such as to have both nondimensional parameters $\eta=M=1$. However, this choice has several drawbacks. The influence of the total mass on the solution is somewhat obscured; and $\bar{x}$ can be very different from the actual scale of the physical domain $\Omega$. Therefore, it may appear necessary to change the scale again in order to perform efficient numerical simulations. For our purpose, it is preferable to choose $\bar{x}$ as a typical length scale of the physical domain $\Omega$, and $\bar{n}$ such as to have $\eta=1$. The parameter $M$ (still called the "mass") will be proportional to the actual number of particles.

Finally, we split the rescaled potential energy as $\delta V=\phi[f]-\phi_{\mathrm{e}}$. The external (or confining) potential $\phi_{\mathrm{e}}$ contains the contributions of the neutralising background and/or the applied voltage:

$$
\begin{aligned}
& -\Delta \phi_{\mathrm{e}}=n_{\mathrm{e}} \quad \text { in } \Omega, \\
& \phi_{\mathrm{e}}=0 \text { on } \Gamma_{1}, \quad \phi_{\mathrm{e}}=\phi_{\mathrm{in}}:=-\delta V_{\mathrm{in}} \text { on } \Gamma_{2}, \quad \partial_{\nu} \phi_{\mathrm{e}}=0 \text { on } \Gamma_{3} .
\end{aligned}
$$

From now on, we shall always consider derivatives with respect to the space variable; therefore we drop the subscript ${ }_{x}$ in $\nabla, \Delta$. What is more, we assume for the sake of simplicity that $\Gamma_{1}$ is not empty (but $\Gamma_{2}$ and $\Gamma_{3}$ can be).

The self-consistent potential $\phi[f]$ is created by the particles under consideration only and satisfies homogeneous boundary conditions everywhere:

$$
-\Delta \phi[f]=\int f \mathrm{~d} \boldsymbol{v} \text { in } \Omega ; \quad \phi[f]=0 \text { on } \Gamma_{1} \cup \Gamma_{2}, \quad \partial_{\nu} \phi[f]=0 \text { on } \Gamma_{3} .
$$

More precisely, we define the linear mappings $\rho, \Phi$ and $\phi$ as follows. The operator $\rho$ : $L^{1}\left(\Omega \times \mathbb{R}^{N}\right) \rightarrow L^{1}(\Omega)$ corresponds to the integration in the variable $\boldsymbol{v}$ :

$$
\forall g \in L^{1}\left(\Omega \times \mathbb{R}^{N}\right), \quad \forall \boldsymbol{x} \in \Omega, \quad \rho[g](\boldsymbol{x}):=\int_{\mathbb{R}^{N}} g(\boldsymbol{x}, \boldsymbol{v}) \mathrm{d} \boldsymbol{v} .
$$

Then, we introduce the space:

$$
V=\left\{v \in H^{1}(\Omega): v=0 \text { on } \Gamma_{1} \cup \Gamma_{2}\right\},
$$

endowed (thanks to the Poincaré inequality) with the norm $\|v\|_{V}:=\|\nabla v\|_{L^{2}(\Omega)}$. The operator $\Phi: V^{\prime} \rightarrow V$ is the inverse of the Laplacian with homogeneous Dirichlet (or 
Dirichlet-Neumann) boundary conditions: given $g \in V^{\prime}, \Phi[g]:=u$ is the solution to the variational formulation:

$$
\int_{\Omega} \nabla u \cdot \nabla v \mathrm{~d} \boldsymbol{x}=\langle g, v\rangle_{V^{\prime}, V}, \quad \forall v \in V .
$$

If $g \in \mathcal{D}^{\prime}(\Omega) \cap V^{\prime}$, then $\Phi[g]$ satisfies the homogeneous Neumann condition on $\Gamma_{3}$. Finally, $\phi:=\Phi \circ \rho$ whenever this mapping is defined. The operators $\Phi$ and $\phi$ are self-adjoint; one notices for instance the formula:

$$
\int_{\Omega} \int_{\mathbb{R}^{N}} f \phi[g] \mathrm{d} \boldsymbol{v} \mathrm{d} \boldsymbol{x}=\int_{\Omega} \int_{\mathbb{R}^{N}} \phi[f] g \mathrm{~d} \boldsymbol{v} \mathrm{d} \boldsymbol{x}=\int_{\Omega} \nabla \phi[f] \cdot \nabla \phi[g] \mathrm{d} \boldsymbol{x},
$$

valid as soon as $\rho[f]$ and $\rho[g] \in V^{\prime}$. By the same token, $\Phi$ achieves an isometry between $V^{\prime}$ and $V$ if the latter is endowed with the above $\|v\|_{V}$ norm and the former with the dual norm.

All in all, the rescaled model (2.6)-(2.8) can be rewritten as:

$$
f(\boldsymbol{x}, \boldsymbol{v})=\gamma\left(\frac{1}{2}|\boldsymbol{v}|^{2}+\phi[f](\boldsymbol{x})-\phi_{\mathrm{e}}(\boldsymbol{x})-\beta\right), \quad \text { with: } \quad \int_{\Omega} \int_{\mathbb{R}^{N}} f \mathrm{~d} \boldsymbol{v} \mathrm{d} \boldsymbol{x}=M,
$$

where the function $\phi_{\mathrm{e}}$ is given by (2.9)-(2.10).

\section{Existence and uniqueness}

\subsection{Assumptions}

Throughout this paper, we shall use the following hypotheses. The domain $\Omega \subset \mathbb{R}^{N}$ is bounded, with a Lipschitz boundary. Furthermore: ${ }^{\mathrm{c}}$

(H1) The solution $\phi_{\mathrm{e}}$ to $(2.9)-(2.10)$ belongs to $L^{\infty}(\Omega) .^{\mathrm{d}}$

(H2) The function $\gamma$ is continuous, with one of the following monotonicity properties (see Figure 1):

(a) either, $\gamma$ is strictly decreasing from $\mathbb{R}$ to $(0,+\infty)$,

(b) or, it is strictly decreasing from $\left(-\infty, s_{*}\right]$ to $[0,+\infty)$, for some $s_{*} \in \mathbb{R}$, and equal to 0 on $\left[s_{*},+\infty\right)$.

Furthermore, it satisfies the following integrability properties:

(1) for all $r \in \mathbb{R}$, the function $s \mapsto s^{\frac{N}{2}-1} \gamma(s+r)$ is integrable on $(0,+\infty)$;

(2) the function $r \mapsto \int_{0}^{+\infty} s^{\frac{N}{2}-1} \gamma(s+r) \mathrm{d} s$ is integrable in a neighborhood of $+\infty$, hence on any interval $(a,+\infty)$.

(H3) Let $\sigma$ be the function $r \mapsto-\int_{0}^{r} \gamma_{*}^{(-1)}(s) \mathrm{d} s$, where $\gamma_{*}^{(-1)}$ is the inverse of $\gamma$ on $(0,+\infty)$, extended by $\gamma_{*}^{(-1)}(0)=s_{*}$ in case (b). We assume:

$$
\lim _{s \rightarrow+\infty} \frac{\sigma(s)}{s}=+\infty \quad \text { and } \quad \forall r \in \mathbb{R}, \quad s \mapsto s^{\frac{N}{2}-1} \sigma(\gamma(s+r))^{-} \in L^{1}\left(\mathbb{R}^{+}\right) .
$$

\footnotetext{
${ }^{\mathrm{c}}$ The existence results of $\S 3$ can be proved under slightly less restrictive assumptions, when the external potential $\phi_{\mathrm{e}}$ is fixed. The framework of (H1)-(H3) is convenient when the external potential is allowed to vary, and in order to prove the estimates of $\S 5$.

${ }^{\mathrm{d}}$ Sufficient conditions to achieve this in the two-dimensional case will be examined in $\S 4$.
} 


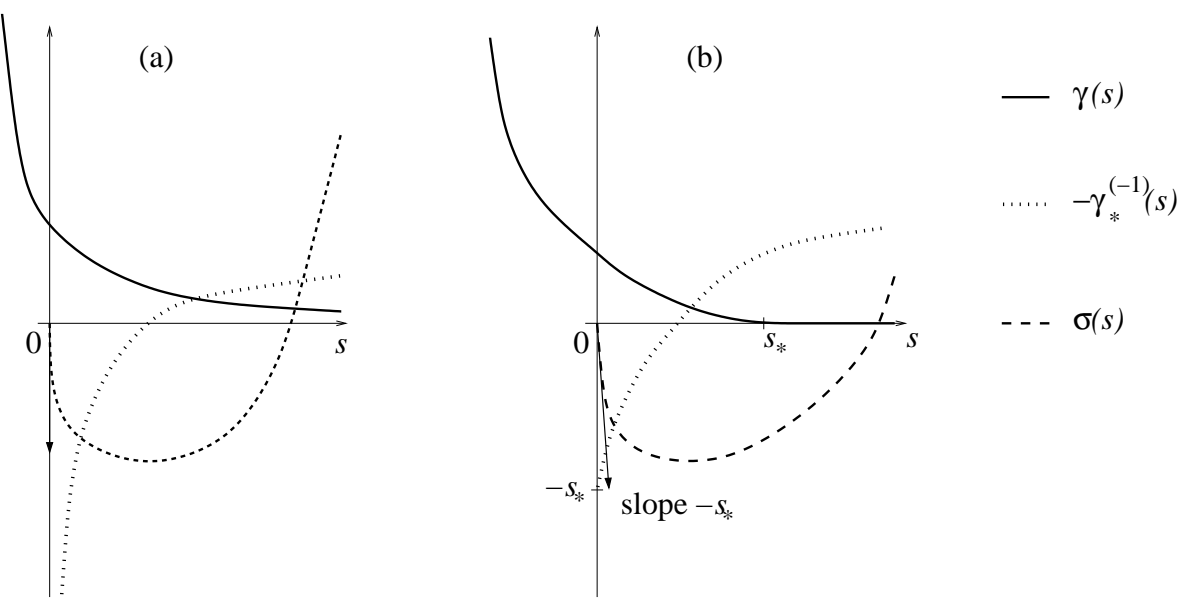

Fig. 1. The functions $\gamma$ and $\sigma$, cases (a) and (b).

By $(\mathrm{H} 2), \sigma$ is strictly convex on $(0,+\infty)$ and belongs to $C^{1}((0,+\infty)) \cup C^{0}([0,+\infty))$. One readily checks that, in case (b), $\sigma$ admits a right derivative $\sigma^{\prime}(0)=-s_{*}$, while in case (a), the right derivative is infinite: $\lim _{s \backslash 0} \sigma(s) / s=-\infty$, cf. Figure 1. The first assumption in (H3) implies that $\sigma$ is bounded from below.

As a consequence of (H1) and item (1) of (H2), one checks ${ }^{2}$ that the function

$$
g:(\boldsymbol{x}, \boldsymbol{v}) \in \Omega \times \mathbb{R}^{N} \mapsto \gamma\left(\frac{1}{2}|\boldsymbol{v}|^{2}-\phi_{\mathrm{e}}(\boldsymbol{x})\right)
$$

belongs to $L^{1}\left(\Omega \times \mathbb{R}^{N}\right)$. The second part of (H3) is automatically satisfied in case (b), as there holds: $\sigma(s)^{-} \leq s_{*} s$. In both cases, it implies $\sigma(g)^{-} \in L^{1}\left(\Omega \times \mathbb{R}^{N}\right)$, allowing one to define $\int_{\Omega \times \mathbb{R}^{N}} \sigma(g)$ as an element of $\mathbb{R} \cup\{+\infty\}$.

\subsection{Kinetic approach}

To prove the existence of a unique solution to Problem (2.15), we use an argument similar to that of Refs. 2, 5, 8. Let

$$
T \Omega:=\Omega \times \mathbb{R}^{N} ; \quad L_{+}^{1}(T \Omega):=\left\{u \in L^{1}(T \Omega): u \geq 0 \text { a.e. }\right\} ;
$$

this is a convex and closed subset of $L^{1}(T \Omega)$. We introduce the functional $\mathcal{J}$ on $L_{+}^{1}(T \Omega)$, defined by

$$
\mathcal{J}[u]:=\underbrace{\int_{T \Omega}\left(\sigma(u)+\left(\frac{1}{2}|\boldsymbol{v}|^{2}-\phi_{\mathrm{e}}\right) u\right) \mathrm{d} \boldsymbol{x} \mathrm{d} \boldsymbol{v}}_{\mathcal{J}_{1}[u]}+\underbrace{\frac{1}{2} \int_{\Omega}|\nabla \phi[u]|^{2} \mathrm{~d} \boldsymbol{x}}_{\mathcal{J}_{2}[u]} .
$$

Remark 3.1. If $\rho[u] \in L^{1}(\Omega) \backslash V^{\prime}$ then $\phi[u]$ does not exist, and in this case, we agree that $\mathcal{J}_{2}[u]=+\infty$. Indeed, by density there exists a sequence $\rho_{n} \in L^{1}(\Omega) \cap V^{\prime}$ 
such that $\rho_{n}$ converges to $\rho[u]$ in $L^{1}(\Omega)$. Then, as $n \rightarrow \infty$ we have

$$
\int_{\Omega}\left|\nabla \Phi\left[\rho_{n}\right]\right|^{2}=\left\|\rho_{n}\right\|_{V^{\prime}}^{2} \rightarrow+\infty
$$

otherwise there exists a subsequence converging weakly in $V^{\prime}$, which would imply $\rho[u] \in V^{\prime}$, a contradiction. Similarly, we agree that

$$
\mathcal{J}_{1}[u]=+\infty \quad \text { if } \quad \sigma(u)+\left(\frac{1}{2}|\boldsymbol{v}|^{2}-\phi_{\mathrm{e}}\right) u \notin L^{1}(T \Omega) ;
$$

we shall see in the proof of Proposition 3.2 that the negative part of this function is integrable for all $u \in L_{+}^{1}(T \Omega)$, under the assumptions of $\S 3.1$. Finally, we extend $\mathcal{J}$ by $+\infty$ on $L^{1}(T \Omega) \backslash L_{+}^{1}(T \Omega)$.

We now study the differentiability of the functional $\mathcal{J}$. Using the property (2.14), we calculate the "formal" derivative:

$$
\mathcal{J}^{\prime}[u]=-\gamma_{*}^{(-1)}(u)+\frac{1}{2}|\boldsymbol{v}|^{2}-\phi_{\mathrm{e}}+\phi[u] .
$$

Proposition 3.1. Let $u \in L_{+}^{1}(T \Omega)$ be such that $\mathcal{J}[u]$ is finite. The subdifferential $\partial \mathcal{J}[u]$ is not empty if, and only if, $\mathcal{J}^{\prime}[u]$ is bounded from below on $T \Omega$, and from above where $u>0$. If these conditions are achieved, the subdifferential is equal to

$$
\partial \mathcal{J}[u]=\left\{\mathcal{J}^{\prime}[u]+\varphi: \varphi \leq 0 \text { and } u \varphi=0 \text { and } \mathcal{J}^{\prime}[u]+\varphi \in L^{\infty}(T \Omega)\right\} .
$$

Proof. Fix $u \in L_{+}^{1}(T \Omega)$, with $\mathcal{J}[u]$ finite. Any $p_{0} \in L^{\infty}(T \Omega)$ belongs to $\partial \mathcal{J}[u]$ iff:

$$
\forall v \in L^{1}(T \Omega), \forall t \in \mathbb{R}, \quad \mathcal{J}[u+t v] \geq \mathcal{J}[u]+t\left\langle p_{0}, v\right\rangle
$$

see Proposition 3, p. 187 of Ref. 1. In practice, it suffices to have this for $v \in$ $T_{u} L_{+}^{1}(T \Omega)$, the tangent cone to $L_{+}^{1}(T \Omega)$ at $u$, otherwise the the left-hand side is infinite. The tangent cone is $T_{u} L_{+}^{1}(T \Omega)=\left\{v \in L^{1}(T \Omega): v \geq 0\right.$ in $\left.\mathcal{A}\right\}=L^{1}(\mathcal{B}) \oplus$ $L_{+}^{1}(\mathcal{A})$, where we have set:

$$
\mathcal{A}:=[u=0]:=\{(\boldsymbol{x}, \boldsymbol{v}) \in T \Omega: u(\boldsymbol{x}, \boldsymbol{v})=0\} \quad \text { and } \quad \mathcal{B}:=[u>0] .
$$

Then, we introduce the following sets of bounded functions with compact support:

$$
\begin{aligned}
& \mathcal{E}_{u}:=\left\{v \in L_{c}^{\infty}(T \Omega): \exists c_{*}, c^{*} \text { s.t. } 0<c_{*} \leq u \leq c^{*}<+\infty \text { on } \operatorname{supp} v\right\} ; \\
& \mathcal{C}_{u}:=\left\{v \in L_{c}^{\infty}(T \Omega): v \geq 0 \text { in } \mathcal{A}, \text { and } v=0 \text { in } \mathcal{B}\right\} .
\end{aligned}
$$

By standard density and truncation arguments, the vector space $\mathcal{E}_{u}$ is dense within $L^{1}(\mathcal{B})$, and $\mathcal{C}_{u}$ is dense within $L_{+}^{1}(\mathcal{A})$; thus $\mathcal{E}_{u} \oplus \mathcal{C}_{u}$ is dense within $T_{u} L_{+}^{1}(T \Omega)$.

For any $v \in L^{1}(T \Omega)$, consider the function $t \mapsto \mathcal{J}[u+t v]$, for $t \in \mathbb{R}$. Differentiating under the integral sign, one sees that if the following conditions are satisfied:

$$
\left(\exists t_{0}(v)>0 \text { s.t. } \forall t \in\left[0, t_{0}(v)\right], \mathcal{J}[u+t v]<+\infty\right) \text { and } \mathcal{J}^{\prime}[u] v \in L^{1}(T \Omega),
$$


then $\mathcal{J}[u+t v]$ admits a right derivative at $t=0$, equal to $\int_{T \Omega} \mathcal{J}^{\prime}[u] v$. As a consequence, for $0 \leq t \leq t_{0}(v)$ there holds:

$$
\mathcal{J}[u+t v]=\mathcal{J}[u]+t \int_{T \Omega} \mathcal{J}^{\prime}[u] v+o(t) .
$$

It is not difficult to check that, for any $v \in \mathcal{E}_{u}$, the conditions (3.6) are satisfied. Furthermore, $-v \in \mathcal{E}_{u}$, meaning that (3.7) holds for $-t_{0}(v) \leq t \leq t_{0}(v)$. Comparing with (3.5), we arrive at:

$$
\left\langle p_{0}, v\right\rangle=\int_{T \Omega} \mathcal{J}^{\prime}[u] v, \quad \forall p_{0} \in \partial \mathcal{J}[u], \quad \forall v \in \mathcal{E}_{u} .
$$

Thanks to the density of $\mathcal{E}_{u}$ within $L^{1}(\mathcal{B})$, we infer $\left.p_{0}\right|_{\mathcal{B}}=\left.\mathcal{J}^{\prime}[u]\right|_{\mathcal{B}}$ if the latter belongs to $L^{\infty}(\mathcal{B})$, otherwise we get a contradiction.

Similarly, we check that (3.6) holds for $v \in \mathcal{C}_{u}$. From (3.5), we deduce:

$$
\left\langle p_{0}, v\right\rangle-\int_{T \Omega} \mathcal{J}^{\prime}[u] v \leq 0, \quad \forall p_{0} \in \partial \mathcal{J}[u], \quad \forall v \in \mathcal{C}_{u},
$$

which implies $\left.\left(p_{0}-\mathcal{J}^{\prime}[u]\right)\right|_{\mathcal{A}} \leq 0$ a.e. in $\mathcal{A}$. If $\mathcal{J}^{\prime}[u]$ is not bounded from below on $\mathcal{A}$, this entails a contradiction, hence $\partial \mathcal{J}[u]=\emptyset$. Otherwise, we have proved $\left.p_{0}\right|_{\mathcal{A}}=\left.\mathcal{J}^{\prime}[u]\right|_{\mathcal{A}}+\varphi$, where $\varphi$ is a non-positive function on $\mathcal{A}$.

Conversely, the same calculations show that any function $p_{0}=\mathcal{J}^{\prime}[u]+\varphi$, where $\varphi=0$ on $\mathcal{B}$ and $\varphi \leq 0$ on $\mathcal{A}$ satisfies the inequality (3.5) for $v \in \mathcal{E}_{u} \oplus \mathcal{C}_{u}$. By density, this extends to $v \in T_{u} L_{+}^{1}(T \Omega)$. Hence, $p_{0} \in \partial \mathcal{J}[u]$ as soon as it is bounded, which means that $\mathcal{J}^{\prime}[u]$ is bounded from below on $\mathcal{B} \cup \mathcal{A}=T \Omega$, and from above on $\mathcal{B}$.

Finally, we consider the following closed convex subset of $L^{1}(T \Omega)$ :

$$
K_{M}(T \Omega):=\left\{u \in L^{1}(T \Omega): u \geq 0 \text { a.e. and } \int_{T \Omega} u \mathrm{~d} \boldsymbol{x} \mathrm{d} \boldsymbol{v}=M\right\} .
$$

Proposition 3.2. There exists a unique function $f$ solution to Problem (2.15), which is the unique minimum of the functional $\mathcal{J}$ on $K_{M}(T \Omega)$.

Proof. First, we establish the existence and uniqueness of the minimum. To begin with, $\mathcal{J}$ is strictly convex; any critical point is a strict and global minimum, hence it is unique. The existence proof is as follows. Using the convexity inequality $\sigma(s)-$ $\sigma(r)-(s-r) \sigma^{\prime}(r) \geq 0$ for any $r, s \geq 0$, and Hypotheses (H1), (H2) and (H3), we have for any non-negative function $u$ :

$$
\begin{aligned}
\sigma(u)+\left(\frac{1}{2}|\boldsymbol{v}|^{2}-\phi_{\mathrm{e}}\right) u & \geq \sigma(g)+\left(\frac{1}{2}|\boldsymbol{v}|^{2}-\phi_{\mathrm{e}}\right) g \\
& \geq \min (\sigma(g), 0)-\left(\left\|\phi_{\mathrm{e}}\right\|_{L^{\infty}}\right) g \in L^{1}(T \Omega),
\end{aligned}
$$

see Eq. (3.1). As the integrand is greater or equal to a fixed integrable function, $\mathcal{J}_{1}[u]$ is bounded from below. Moreover, a standard argument (see Ref. 1, p. 13) shows that $\mathcal{J}_{1}$ is lower semi-continuous on $L_{+}^{1}(T \Omega)$. On the other hand, $\mathcal{J}_{2}$ is non-negative, and its lower semi-continuity is proved by an argument similar to Remark 3.1. 
All together, we see that $\mathcal{J}=\mathcal{J}_{1}+\mathcal{J}_{2}$ is bounded from below and lower semicontinuous, hence weakly l.s.c. on $L_{+}^{1}(T \Omega)$, a fortiori on $K_{M}(T \Omega):=K$. Furthermore, taking $u$ as a non-negative bounded function with compact support on $T \Omega$ and mass $M$, one easily checks that $\mathcal{J}[u]$ is finite. Therefore, $\mathcal{J}$ has a finite infimum on $K$.

Let $\left(f_{n}\right)_{n} \in K$ be a minimizing sequence for $\mathcal{J}$. Using (H3) and the DunfordPettis compactness criterion, we can extract a subsequence still denoted $\left(f_{n}\right)$ which converges weakly to $f \in L^{1}(T \Omega)$. As $K$ is convex and closed, it is weakly closed, and $f \in K$. And, since $\mathcal{J}$ is weakly l.s.c., then

$$
\mathcal{J}[f] \leq \liminf _{n \rightarrow \infty} \mathcal{J}\left[f_{n}\right]=\inf _{K} \mathcal{J},
$$

meaning that $f$ is the global minimum of $\mathcal{J}$. As a consequence (see Ref. 1, p. 189):

$$
0 \in \partial \mathcal{J}[f]+N_{f} K
$$

where $N_{f} K$ is the normal cone to $K$ at $f$, i.e., the negative polar cone to the tangent cone $T_{f} K$. For any $u \in K$, we calculate the tangent and normal cones:

$$
\begin{aligned}
T_{u} K= & \left\{v \in L^{1}(T \Omega): \int_{T \Omega} v=0 \text { and } v \geq 0 \text { where } u=0\right\} ; \\
N_{u} K= & \{c+\psi\} \subset L^{\infty}(T \Omega), \quad \text { where: } \\
& c=\text { const. }, \quad 0 \geq \psi \in L^{\infty}(T \Omega), \quad u \psi=0 .
\end{aligned}
$$

The optimality condition (3.8) implies that $\mathcal{J}^{\prime}[f]$ is not empty, so it is described by (3.4), and we arrive at $\mathcal{J}^{\prime}[f]-\beta+\nu=0$, where $\beta \in \mathbb{R}$ and $\nu:=\varphi+\psi \leq 0$ satisfies $f \nu=0$. Using the formula (3.3) and noting that $\gamma\left(\gamma_{*}^{(-1)}(s)\right)=s$ for all $s \geq 0$, this means:

- where $f>0, f(\boldsymbol{x}, \boldsymbol{v})=\gamma\left(\frac{1}{2}|\boldsymbol{v}|^{2}+\phi[f](\boldsymbol{x})-\phi_{\mathrm{e}}(\boldsymbol{x})-\beta\right)$;

- where $f=0, f(\boldsymbol{x}, \boldsymbol{v})=\gamma\left(\frac{1}{2}|\boldsymbol{v}|^{2}+\phi[f](\boldsymbol{x})-\phi_{\mathrm{e}}(\boldsymbol{x})-\beta+\nu(\boldsymbol{x}, \boldsymbol{v})\right)$.

As $\gamma$ is non-increasing and non-negative, this implies:

$$
f(\boldsymbol{x}, \boldsymbol{v})=0 \geq \gamma\left(\frac{1}{2}|\boldsymbol{v}|^{2}+\phi[f](\boldsymbol{x})-\phi_{\mathrm{e}}(\boldsymbol{x})-\beta\right) \geq 0=f(\boldsymbol{x}, \boldsymbol{v}) .
$$

Therefore, $f$ is a solution to $(2.15)$.

As $\gamma$ is bijective (one-to-one and onto) whenever it is strictly positive, the uniqueness of $f$ implies that of $\beta$. This allows one to take the following definition.

Definition 3.1. Fix $\phi_{\mathrm{e}} \in L^{\infty}(\Omega)$. The mapping $\mathcal{S}: \mathbb{R}_{*}^{+} \rightarrow \mathbb{R}$ is given by $\mathcal{S}(M)=\beta$, the multiplier which appears in the solution to Problem (2.15). 


\subsection{Potential approach}

As noted in the Introduction, it is fruitful to look at Problem (2.15) from the point of view of the potential $\phi[f]$, rather than $f$. To this end, we introduce the function $G$ defined as:

$$
G(r)=\int_{\mathbb{R}^{N}} \gamma\left(\frac{1}{2}|\boldsymbol{v}|^{2}+r\right) \mathrm{d} \boldsymbol{v}=2^{\frac{N}{2}-1}\left|S^{N-1}\right| \int_{0}^{+\infty} s^{\frac{N}{2}-1} \gamma(s+r) \mathrm{d} s ;
$$

where $\left|S^{N-1}\right|$ is the $(N-1)$-dimensional area of the unit sphere of $\mathbb{R}^{N}$; we have used a change of variables in polar coordinates. The finiteness of $G(r)$ for all $r$ follows from (H2). If $f$ is solution to Problem (2.15), then $\phi:=\phi[f]$ solves:

$$
-\Delta \phi=G\left(\phi-\phi_{\mathrm{e}}-\beta\right):=\rho \text { in } \Omega, \quad \phi=0 \text { on } \Gamma_{1} \cup \Gamma_{2}, \quad \partial_{\nu} \phi=0 \text { on } \Gamma_{3} .
$$

where $\beta=\mathcal{S}(M)$, see Definition 3.1. Notice that $\gamma$ being non-increasing implies that $G$ is non-increasing.

Proposition 3.3. For any $\phi_{\mathrm{e}} \in L^{\infty}(\Omega)$ and $\beta \in \mathbb{R}$, there exists a unique solution to Problem (3.10).

Proof. The variational formulation of this problem writes:

Find $\phi \in V$ such that,

$$
\int_{\Omega} \nabla \phi \cdot \nabla \xi=\int_{\Omega} G\left(\phi-\phi_{\mathrm{e}}-\beta\right) \xi, \quad \forall \xi \in V .
$$

Clearly, $\phi$ is a solution to (3.11) iff it is a critical point of the functional $\mathcal{F}: V \longrightarrow \mathbb{R}$ defined by

$$
\mathcal{F}[\phi]=\frac{1}{2} \int_{\Omega}|\nabla \phi|^{2}+\int_{\Omega} \mathcal{G}\left(\phi-\phi_{\mathrm{e}}-\beta\right)
$$

where $\mathcal{G}(r):=\int_{r}^{+\infty} G(s) \mathrm{d} s$; this function is well-defined by (H2). As $\mathcal{G}$ is positive, we see that $\mathcal{F}$ is coercive. Furthermore, $G$ being decreasing implies that $\mathcal{G}$ is convex. Thus, a standard argument shows that the functional $\mathcal{F}$ is strictly convex and (weakly) l.s.c. Furthermore, it is not identically $+\infty$, as $\mathcal{F}[0]$ is finite by (H1) and (H2). This ensures the existence and uniqueness of the critical point.

Definition 3.2. Fix $\phi_{\mathrm{e}} \in L^{\infty}(\Omega)$. For any $\beta \in \mathbb{R}$, let $\phi$ be the solution to Problem (3.10). The mapping $\mu: \mathbb{R} \rightarrow \mathbb{R}_{*}^{+}$is given by:

$$
\mu(\beta):=\int_{\Omega} G\left(\phi-\phi_{\mathrm{e}}-\beta\right) \mathrm{d} \boldsymbol{x} .
$$

From the above discussion, it follows that $\mu(\mathcal{S}(M))=M$, for all $M>0$. Conversely, let $\beta \in \mathbb{R}$ be given. Set $\phi$ as the solution to Problem (3.10), and $M=\mu(\beta)$. Then, define $\tilde{f}(\boldsymbol{x}, \boldsymbol{v}):=\gamma\left(\frac{1}{2}|\boldsymbol{v}|^{2}+\phi(\boldsymbol{x})-\phi_{\mathrm{e}}(\boldsymbol{x})-\beta\right)$. Integrating in $\boldsymbol{v}$, we find:

$$
\rho[\tilde{f}]=G\left(\phi-\phi_{\mathrm{e}}-\beta\right), \quad \text { i.e. } \quad-\Delta \phi[\tilde{f}]=-\Delta \phi .
$$


As $\phi$ and $\phi[\tilde{f}]$ satisfy the same boundary conditions, they are equal. Hence, $\tilde{f}$ is the (unique) solution to Problem (2.15) for the mass $M$; in other words, $\beta=\mathcal{S}(M)$. So, $\mathcal{S}(\mu(\beta))=\beta$, for all $\beta \in \mathbb{R}$. Summarising, we have:

Proposition 3.4. Fix $\phi_{\mathrm{e}} \in L^{\infty}(\Omega)$. The mappings $\mathcal{S}$ and $\mu$, as in Definitions 3.1 and 3.2 respectively, are bijective, and inverse of each other.

This proves the equivalence of Problems (2.15) and (3.10).

\subsection{Monotonicity and regularity properties}

Now, we study the dependence of the solution to (3.10) with respect to the data, and the regularity of the solutions to (3.10) and (2.15). First, the monotonicity of the function $G$ entails a comparison principle. ${ }^{19}$

Proposition 3.5. Let $\phi_{1}$ and $\phi_{2}$ be two solutions to Problem (3.10) corresponding to $\left(\phi_{\mathrm{e}}, \beta\right)=\left(\phi_{\mathrm{e}}^{1}, \beta_{1}\right)$ and $\left(\phi_{\mathrm{e}}^{2}, \beta_{2}\right)$ respectively. If $\phi_{\mathrm{e}}^{1}+\beta_{1} \geq \phi_{\mathrm{e}}^{2}+\beta_{2}$ a.e. in $\Omega$, then $\phi_{1} \geq \phi_{2}$ in $\Omega$.

It is also possible to compare the "masses" associated to different instances of Problem (3.10).

Proposition 3.6. Under the hypotheses of Proposition 3.5, define for $i=1,2$ :

$$
\rho_{i}:=-\Delta \phi_{i}=G\left(\phi_{i}-\phi_{\mathrm{e}}^{i}-\beta_{i}\right) \quad \text { and } \quad M_{i}=\int_{\Omega} \rho_{i} .
$$

If $\phi_{\mathrm{e}}^{1}+\beta_{1} \geq \phi_{\mathrm{e}}^{2}+\beta_{2}$ a.e. in $\Omega$, then $M_{1} \geq M_{2}$.

Proof. By the previous Proposition, we decompose $\Omega=\left[\phi_{1}=\phi_{2}\right] \cup\left[\phi_{1}>\phi_{2}\right]=$ $\mathcal{A} \cup \mathcal{B}$. The function $w=\phi_{1}-\phi_{2}$ is solution to

$$
\int_{\Omega} \nabla w \cdot \nabla \xi=\int_{\Omega}\left(\rho_{1}-\rho_{2}\right) \xi, \quad \forall \xi \in V .
$$

Let us take $\xi=\Upsilon_{\epsilon}(w)$ as a test function in the previous formulation, where $\Upsilon_{\epsilon}(\cdot)$ is defined as

$$
\Upsilon_{\epsilon}(r)=1 \text { if } r \geq \epsilon, \quad \Upsilon_{\epsilon}(r)=r / \epsilon \text { if } 0 \leq r \leq \epsilon, \quad \Upsilon_{\epsilon}(r)=0 \text { if } r \leq 0 .
$$

We arrive at:

$$
\int_{\Omega}|\nabla w|^{2} \Upsilon_{\epsilon}^{\prime}(w)=\int_{\Omega}\left(\rho_{1}-\rho_{2}\right) \Upsilon_{\epsilon}(w) .
$$

The left-hand side of this equation is non-negative. When $\epsilon$ tends to $0, \Upsilon_{\epsilon}(w)$ converges pointwise toward the Heaviside function $\Upsilon(w)$, with the convention $\Upsilon(0)=0$. Therefore, by the dominated convergence theorem, we get:

$$
\int_{\Omega}\left(\rho_{1}-\rho_{2}\right) \Upsilon(w) \geq 0, \quad \text { i.e. } \quad \int_{\mathcal{B}} \rho_{1} \geq \int_{\mathcal{B}} \rho_{2} .
$$


On the other hand, on the set $\mathcal{A}$ there holds:

$$
\phi_{1}-\phi_{\mathrm{e}}^{1}-\beta^{1} \leq \phi_{2}-\phi_{\mathrm{e}}^{2}-\beta^{2}, \quad \text { hence } G\left(\phi_{1}-\phi_{\mathrm{e}}^{1}-\beta^{1}\right) \geq G\left(\phi_{2}-\phi_{\mathrm{e}}^{2}-\beta^{2}\right),
$$

and $\int_{\mathcal{A}} \rho_{1} \geq \int_{\mathcal{A}} \rho_{2}$. Adding the two contributions, we find $M_{1} \geq M_{2}$.

Corollary 3.1. Let $\phi_{\mathrm{e}} \in L^{\infty}(\Omega)$ be given. The mappings $\mathcal{S}$ and $\mu$, as in Definitions 3.1 and 3.2 respectively, are strictly increasing.

Proof. As a consequence of the previous Proposition, $\mu$ is non-decreasing. As a bijection between real intervals (Proposition 3.4), it must then be strictly increasing; and the same holds for its inverse mapping $\mathcal{S}$.

Similarly, one may study the dependence of $\phi$ on parameters other than $M$, through the external potential $\phi_{\mathrm{e}}$, as we shall do in $\S 5.2$.

Corollary 3.2. Consider two bounded external potentials $\phi_{\mathrm{e}}^{1} \geq \phi_{\mathrm{e}}^{2}$. Let $f_{i}, i=1,2$ be the solutions to (2.15) with $\phi_{\mathrm{e}}=\phi_{\mathrm{e}}^{i}$ and the same mass $M>0$, and $\beta_{i}$ the corresponding multipliers. Then, $\beta_{1} \leq \beta_{2}$.

Proof. The potentials $\phi_{i}=\phi\left[f_{i}\right]$ satisfy:

$$
-\Delta \phi_{i}=G\left(\phi_{i}-\phi_{\mathrm{e}}^{i}-\beta_{i}\right), \quad \phi_{i}=0 \text { on } \Gamma_{1} \cup \Gamma_{2}, \quad \partial_{\nu} \phi_{i}=0 \text { on } \Gamma_{3}, \quad \text { for } i=1,2 .
$$

Introducing the solution $\tilde{\phi}$ to

$$
\begin{aligned}
& -\Delta \tilde{\phi}=G\left(\tilde{\phi}-\phi_{\mathrm{e}}^{2}-\beta_{1}\right), \quad \tilde{\phi}=0 \text { on } \Gamma_{1} \cup \Gamma_{2}, \quad \partial_{\nu} \tilde{\phi}=0 \text { on } \Gamma_{3}, \\
& \text { and } \quad \tilde{M}=\int_{\Omega} G\left(\tilde{\phi}-\phi_{\mathrm{e}}^{2}-\beta_{1}\right) \mathrm{d} \boldsymbol{x},
\end{aligned}
$$

it follows from Propositions 3.5 and 3.6 that $\phi_{1} \geq \tilde{\phi}$, and $M \geq \tilde{M}$. Comparing then $\phi_{2}$ and $\tilde{\phi}$ by Corollary 3.1, one deduces $\beta_{2} \geq \beta_{1}$.

Definition 3.3. Let $p \geq 2 N /(N+2)$ for $N \geq 3$, or $p>1$ for $N=2$. We call $\Phi_{p}$ the space of potentials

$$
\Phi_{p}=\left\{v \in H^{1}(\Omega): \Delta v \in L^{p}(\Omega), v=0 \text { on } \Gamma_{1} \cup \Gamma_{2}, \partial_{\nu} v=0 \text { on } \Gamma_{3}\right\},
$$

i.e., the space of solutions to (2.13) when $g \in L^{p}(\Omega)$. The condition on $p$ implies that $L^{p}(\Omega) \subset V^{\prime}$, and $\|v\|_{\Phi_{p}}:=\|\Delta v\|_{L^{p}(\Omega)}$ defines a norm on $\Phi_{p}$, equivalent to the canonical norm.

Lemma 3.1. Let $f$ be the solution of Problem (2.15). Then $f \in L^{\infty}\left(\Omega \times \mathbb{R}^{N}\right)$, $\rho[f] \in L^{\infty}(\Omega)$ and $\phi[f] \in \Phi_{p}$ for all $p \leq \infty$.

Proof. As $\gamma$ takes its values in $\mathbb{R}^{+}$, we obviously have $f \geq 0$ and $\rho[f] \geq 0$, hence $\phi[f] \geq 0$. Using (H1) and the fact that $\gamma$ is a decreasing function, we have:

$$
f(\boldsymbol{x}, \boldsymbol{v})=\gamma\left(\frac{1}{2}|\boldsymbol{v}|^{2}+\phi[f](\boldsymbol{x})-\phi_{\mathrm{e}}(\boldsymbol{x})-\beta\right) \leq \gamma\left(-\left\|\phi_{\mathrm{e}}\right\|_{\infty}-\beta\right) .
$$


Similarly, $G$ being a decreasing function implies

$$
\rho[f](\boldsymbol{x})=G\left(\phi[f](\boldsymbol{x})-\phi_{\mathrm{e}}(\boldsymbol{x})-\beta\right) \leq G\left(-\left\|\phi_{\mathrm{e}}\right\|_{\infty}-\beta\right) .
$$

Therefore, $\rho[f] \in L^{\infty}(\Omega)$ and $\phi[f] \in \Phi_{p}$, for all $p \leq \infty$.

\section{Corner behavior in dimension 2}

In the rest of the article, we consider the case where $\Omega$ is a polygonal domain of $\mathbb{R}^{2}$, an example of which is shown on Figure 2. If $\boldsymbol{c}$ is any corner on $\partial \Omega$, we denote:

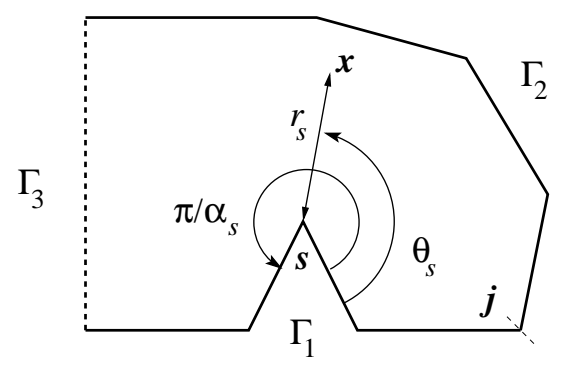

Fig. 2. The polygonal domain $\Omega$.

- $\frac{\pi}{\alpha_{c}}$ (with $\left.\alpha_{c} \in\left(\frac{1}{2},+\infty\right)\right)$ the interior angle at $\boldsymbol{c}$;

- $\left(r_{c}, \theta_{c}\right)$ the local polar coordinates, with $0<\theta_{c}<\pi / \alpha_{c}$;

- $\Gamma_{c}$ the subset of $\partial \Omega$ made of the two sides that meet at $\boldsymbol{c}$;

- $\chi_{c} \in C^{\infty}$ a cut-off function, which depends on $r_{c}$ only, and is supported in a neighborhood $\Omega_{c}$ of $\boldsymbol{c}$ such that of $\partial \Omega_{c} \cap \partial \Omega \subset \Gamma_{c}$.

To begin with, let us recall Grisvard's results ${ }^{11,12}$ on the linear Poisson problem. As noted above, $L^{p}(\Omega) \subset V^{\prime}$ (see (2.12)) for all $p>1$. Let $u$ be the solution to (2.13) for $g \in L^{p}(\Omega)$. Then, for each corner there exists a finite sequence of scalars $\left(\lambda_{\ell}^{c}\right)_{\ell \in \Lambda_{c}^{p}}$ (the singularity coefficients) such that

$$
u-\sum_{c} \sum_{\ell \in \Lambda_{c}^{p}} \lambda_{\ell}^{c} S_{\ell}^{c}:=u_{\mathcal{R}} \in W^{2, p}(\Omega) .
$$

The functions $u_{\mathcal{R}}$ and $\sum_{c} \sum_{\ell \in \Lambda_{c}^{p}} \lambda_{\ell}^{c} S_{\ell}^{c}$ are respectively called the regular and singular parts of $u$. Both depend continuously on $g$, i.e.,

$$
\left\|u_{\mathcal{R}}\right\|_{W^{2, p}(\Omega)}+\sum_{c} \sum_{\ell \in \Lambda_{c}^{p}}\left|\lambda_{\ell}^{c}\right| \leq C(\Omega, p)\|g\|_{L^{p}(\Omega)} .
$$

The indexing set $\Lambda_{c}^{p}$ and the local primal singular functions $S_{\ell}^{c}$ are given by:

- if $\boldsymbol{c}$ is a pure Dirichlet corner $\left(\Gamma_{c} \subset \Gamma_{1} \cup \Gamma_{2}\right)$ :

$$
\Lambda_{c}^{p}:=\mathbb{Z}^{*} \cap\left(\frac{p-2}{p \alpha_{c}}, \frac{2 p-2}{p \alpha_{c}}\right), \quad S_{\ell}^{c}\left(r_{c}, \theta_{c}\right):=\chi_{c}\left(r_{c}\right) r_{c}^{\ell \alpha_{c}} \sin \left(\ell \alpha_{c} \theta_{c}\right)
$$


- if $\boldsymbol{c}$ is a pure Neumann corner $\left(\Gamma_{c} \subset \Gamma_{3}\right)$ :

$$
\Lambda_{c}^{p}:=\mathbb{Z} \cap\left(\frac{p-2}{p \alpha_{c}}, \frac{2 p-2}{p \alpha_{c}}\right), \quad S_{\ell}^{c}\left(r_{c}, \theta_{c}\right):=\chi_{c}\left(r_{c}\right) r_{c}^{\ell \alpha_{c}} \cos \left(\ell \alpha_{c} \theta_{c}\right) ;
$$

- if $\boldsymbol{c}$ is a mixed corner, with a Dirichlet condition on the side $\theta_{c}=0$ and a Neumann condition on the side $\theta_{c}=\pi / \alpha_{c}$ :

$$
\Lambda_{c}^{p}:=\mathbb{Z} \cap\left(\frac{p-2}{p \alpha_{c}}+\frac{1}{2}, \frac{2 p-2}{p \alpha_{c}}+\frac{1}{2}\right), \quad S_{\ell}^{c}\left(r_{c}, \theta_{c}\right):=\chi_{c}\left(r_{c}\right) r_{c}^{\left(\ell-\frac{1}{2}\right) \alpha_{c}} \sin \left(\left(\ell-\frac{1}{2}\right) \alpha_{c} \theta_{c}\right) .
$$

As the singular functions $S_{\ell}^{c}$ are smooth away from $\boldsymbol{c}$, the function $u$ is of $W^{2, p}$ regularity except in a neighborhood of the corners. We shall mainly concentrate on the regularity in the scale $H^{s}(\Omega)$, the most natural one both from a theoretical and a computational point of view, given the semi-linear nature of the problem. From the above description of the singular functions, one checks that a pure Dirichlet or Neumann corner $\boldsymbol{c}$ is singular, i.e., $u_{\Omega_{\Omega_{c}}} \notin H^{2}\left(\Omega_{c}\right)$ in general, iff it is reentrant $\left(\alpha_{c}<1\right)$. A mixed corner $\boldsymbol{c}$ is singular iff it is obtuse or reentrant $\left(\alpha_{c}<2\right)$. To simplify the discussion, we shall assume the following hypothesis in the sequel:

(H4) There is exactly one reentrant corner $\boldsymbol{s}$; it is a pure Dirichlet corner, with $\Gamma_{s} \subset \Gamma_{1}$. All mixed corners (if any) have angles smaller than or equal to $\frac{\pi}{2}$.

Therefore, we shall generally drop the subscript ${ }_{s}$ in $r_{s}, \theta_{s}, \alpha_{s}$, etc. Nevertheless, the general case can be handled in the same manner.

We have the following estimate on the regular part in the neighborhood of the reentrant corner.

Lemma 4.1. Let $g \in L^{p}(\Omega), u=\Phi[g]$, for $p>2$. The regular part $u_{\mathcal{R}}$ satisfies:

$$
\left|\frac{\chi(r) u_{\mathcal{R}}(r, \theta)}{r \sin (\alpha \theta)}\right| \leq C(\Omega, p)\|g\|_{L^{p}(\Omega)} .
$$

Therefore, $u$ is equivalent to its singular part $\lambda \chi(r) r^{\alpha} \sin (\alpha \theta)$ as $\boldsymbol{x} \rightarrow \boldsymbol{s}$.

Proof. Let $w:=\chi u_{\mathcal{R}}$. We have $w \in W^{2, p}(\Omega) \cap W_{0}^{1, p}(\Omega)$, hence $\nabla w \in W^{1, p}(\Omega)^{2} \subset$ $C(\bar{\Omega})^{2}$, and $w$ is supported in $\Omega_{s}$. From (4.2), it follows that

$$
\|\nabla w\|_{L^{\infty}\left(\Omega_{s}\right)} \leq C(\Omega, p)\|\nabla w\|_{W^{1, p}(\Omega)} \leq C(\Omega, p)\|g\|_{L^{p}(\Omega)} .
$$

Of course, this bound applies to the $\theta$-component of $\nabla w$, which is $\frac{1}{r} \frac{\partial w}{\partial \theta}$. As $w$ vanishes on the boundary, we have $\frac{w}{r}(r, 0)=0$ for any $r>0$. Therefore:

$$
\frac{w(r, \theta)}{r}=\int_{0}^{\theta} \frac{\partial}{\partial \tilde{\theta}}\left(\frac{w(r, \tilde{\theta})}{r}\right) \mathrm{d} \tilde{\theta},
$$

\footnotetext{
e The reader will adapt the statement to the opposite conditions.
} 
and

$$
\left|\frac{w(r, \theta)}{r}\right| \leq \theta\left\|\frac{\partial}{\partial \theta}\left(\frac{w}{r}\right)\right\|_{L^{\infty}\left(\Omega_{s}\right)} \leq C \theta\|g\|_{L^{p}(\Omega)}
$$

In the same manner, we have:

$$
\frac{w\left(r, \frac{\pi}{\alpha}\right)}{r}=0, \quad \text { and } \quad\left|\frac{w(r, \theta)}{r}\right| \leq C\left(\frac{\pi}{\alpha}-\theta\right)\|g\|_{L^{p}(\Omega)} .
$$

On the other hand, one easily checks that

$$
\forall \theta \in\left[0, \frac{\pi}{\alpha}\right], \quad \min \left(\theta, \frac{\pi}{\alpha}-\theta\right) \leq \frac{\pi}{2 \alpha} \sin (\alpha \theta) .
$$

This completes the proof of Lemma.

Now we return to the non-linear problems (3.10) and (2.15). We know from Lemma 3.1 that $\phi=\phi[f] \in \Phi_{p}$ for any $p$. The Sobolev imbeddings and the analytic expression of singularities imply the existence of $\epsilon>0$ such that $\Phi_{p} \subset H^{1+\epsilon}(\Omega) \subset$ $C(\bar{\Omega})$ for any polygonal domain. ${ }^{12}$ To study the local behavior of the total potential $V=\phi[f]-\phi_{\mathrm{e}}$ near the reentrant corner, we introduce the following stronger version of (H1), which implies ${ }^{11,12}$ that $\phi_{\mathrm{e}}$ locally belongs to $\Phi_{p}$.

(H1') There is $p>2$ such that $n_{\mathrm{e}} \in L^{p}(\Omega)$ and $\phi_{\text {in }} \in W^{p, 2-1 / p}\left(\Gamma_{2}\right)$.

Furthermore, if $\bar{\Gamma}_{1} \cap \bar{\Gamma}_{2} \neq \emptyset$, there must hold at any $j \in \bar{\Gamma}_{1} \cap \bar{\Gamma}_{2}$ :

$$
\phi_{\text {in }}(\boldsymbol{j})=0 \quad \text { and, unless } \alpha_{j}=2 \text { or } \alpha_{j}=\frac{2}{3}, \quad \partial_{\tau_{2}} \phi_{\text {in }}(j)=0,
$$

where $\boldsymbol{\tau}_{2}$ is the tangent unit vector on $\Gamma_{2}$. The exceptions involve right angles.

Therefore, the regular-singular decomposition of these functions writes:

$$
\phi=\phi_{\mathcal{R}}+\lambda \chi(r) r^{\alpha} \sin (\alpha \theta), \quad \phi_{\mathrm{e}}=\phi_{\mathrm{e}, \mathcal{R}}+\lambda_{\mathrm{e}} \chi(r) r^{\alpha} \sin (\alpha \theta),
$$

with $\phi_{\mathcal{R}}, \phi_{\mathrm{e}, \mathcal{R}} \in W^{2, p}(\Omega)$ for $p<p^{*}$. The upper bound $p^{*}>2$ depends on the geometry of $\Omega$ and on the hypothesis (H1'). ${ }^{\mathrm{f}}$ Furthermore, $\left.\left.\phi_{\mathcal{R}}\right|_{\Omega_{s}} \phi_{\mathrm{e}, \mathcal{R}}\right|_{\Omega_{s}} \in W^{2, p}\left(\Omega_{s}\right)$ for any $p<1 /(1-\alpha)$ (resp. any such $p$ satisfying $n_{\mathrm{e}} \in L^{p}\left(\Omega_{s}\right)$ ). Notice, however, that (H1) is sufficient if one is interested in the behavior of $\phi$ alone. Sufficient (and nearly optimal) conditions to achieve (H1) are:

$$
\begin{aligned}
& n_{\mathrm{e}} \in H^{-1+\epsilon}(\Omega), \quad \phi_{\text {in }} \in H^{1 / 2+\epsilon}\left(\Gamma_{2}\right), \quad \phi_{\text {in }}(\boldsymbol{j})=0, \forall \boldsymbol{j} \in \bar{\Gamma}_{1} \cap \bar{\Gamma}_{2}, \\
\text { or } \quad & n_{\mathrm{e}} \in L^{p_{1}}(\Omega), \quad \phi_{\text {in }} \in W^{1-1 / p_{2}, p_{2}}\left(\Gamma_{2}\right), \quad \phi_{\text {in }}(\boldsymbol{j})=0, \forall \boldsymbol{j} \in \bar{\Gamma}_{1} \cap \bar{\Gamma}_{2},
\end{aligned}
$$

for some $\epsilon>0$, respectively $p_{1}>1$ and $p_{2}>2$.

${ }^{\mathrm{f}}$ Under (H4), the precise conditions are: (i) $\left(1-\alpha_{s}\right) p<1$ for the reentrant corner; (ii) $\left(2-\alpha_{c}\right) p<2$ for the salient $\left(\alpha_{c}>1\right)$ corners with pure Dirichlet or Neumann conditions; (iii) $\left(4-\alpha_{c}\right) p<4$ for the acute $\left(\alpha_{c}>2\right)$ corners with mixed conditions; (iv) as far as the regularity of $\phi_{\mathrm{e}, \mathcal{R}}$ is concerned, $p$ satisfies (H1'). Note that, thanks to a symmetry principle, the mixed-condition rightangle corners are not singular. 
Interestingly, the singularity coefficient of $\phi$ enjoys a monotonicity property.

Theorem 4.1. Let $f_{1}$ and $f_{2}$ be two solutions of Problem (2.15), corresponding to $M=M_{1}$ and $M_{2}$ respectively, with the same external potential $\phi_{\mathrm{e}} \in L^{\infty}(\Omega)$. Let $\lambda_{1}$ and $\lambda_{2}$ be the singularity coefficients of $\phi_{1}:=\phi\left[f_{1}\right]$ and $\phi_{2}:=\phi\left[f_{2}\right]$. If $M_{1} \geq M_{2}$, then $\lambda_{1} \geq \lambda_{2}$.

Proof. Thanks to Lemma 4.1, we have

$$
\phi_{1}-\phi_{2} \sim\left(\lambda_{1}-\lambda_{2}\right) r^{\alpha} \sin (\alpha \theta) \text { as } r \rightarrow 0 \text {, uniformly in } \theta .
$$

Let $\beta_{i}, i=1,2$, be the multipliers associated to $f_{i}$; then $\phi_{i}$ satisfies $-\Delta \phi_{i}=$ $G\left(\phi_{i}-\phi_{\mathrm{e}}-\beta_{i}\right)$. Thanks to Corollary 3.1, we have $\beta_{1} \geq \beta_{2}$, and $\phi_{1} \geq \phi_{2}$ by Proposition 3.3; therefore $\lambda_{1} \geq \lambda_{2}$.

Finally, we recall that $\lambda$ is given by the representation formula: ${ }^{12}$

$$
\lambda=\int_{\Omega} \rho P_{s} \mathrm{~d} \boldsymbol{x}
$$

where the dual singular function $P_{s}$ is characterized by the following properties:

$$
\begin{aligned}
& -\Delta P_{s}=0 \text { in } \Omega, \quad P_{s}=0 \text { on } \Gamma_{1} \cup \Gamma_{2}, \quad \partial_{\nu} P_{s}=0 \text { on } \Gamma_{3} ; \\
& P_{s}=P_{p}+\tilde{P}, \quad \text { where: } \quad P_{p}=\frac{1}{\pi} r^{-\alpha} \sin (\alpha \theta), \quad \tilde{P} \in H^{1}(\Omega) \cap C(\bar{\Omega}) .
\end{aligned}
$$

Thus, $P_{s} \in L^{2}(\Omega)$, and $P_{s} \geq 0$ : a non-negative right-hand side implies a nonnegative potential and thus a non-negative singularity coefficient. As the principal

part $P_{p}$ is zero on $\Gamma_{s}$, and smooth on the other sides of $\partial \Omega$, the remainder $\tilde{P}$ appears as the variational solution to:

$$
-\Delta \tilde{P}=\Delta P_{p}=0 \text { in } \Omega, \quad \tilde{P}=-P_{p} \quad \text { on } \Gamma_{1} \cup \Gamma_{2}, \quad \partial_{\nu} \tilde{P}=-\partial_{\nu} P_{p} \text { on } \Gamma_{3} .
$$

As a consequence, $\tilde{P}$ locally belongs to $\Phi_{p}$ for all $p$, near the reentrant corner $s$. By Lemma 4.1 and (4.8), we infer that there exists $\tilde{\zeta} \in \mathbb{R}$ such that

$$
\tilde{P} \sim \tilde{\zeta} r^{\alpha} \sin (\alpha \theta) \quad \text { and } \quad P_{s} \sim \frac{1}{\pi} r^{-\alpha} \sin (\alpha \theta) \quad \text { as } r \rightarrow 0 \text {, uniformly in } \theta .
$$

\section{Behavior of the Maxwell-Boltzmann Problem}

From now on, we assume $\gamma(s)=\exp (-s)$; one readily checks that $\gamma(s)$ and $\sigma(s)=$ $s \ln s-s$ satisfy the assumptions of $\S 3.1$ : this is the Maxwellian distribution. Then, Eq. (3.9) yields $G(r)=2 \pi \exp (-r)$, and Problems (3.10) or (2.15) become:

$$
\begin{aligned}
& -\Delta \phi=\kappa \exp \left(\phi_{\mathrm{e}}-\phi\right):=\rho \text { in } \Omega, \quad \text { with: } \int_{\Omega} \rho=M, \\
& \phi=0 \text { on } \Gamma_{1} \cup \Gamma_{2}, \quad \partial_{\nu} \phi=0 \text { on } \Gamma_{3} ;
\end{aligned}
$$

where we have set $\kappa=2 \pi \exp (\beta)$. 


\subsection{Behaviour with respect to the mass}

In this section, we fix $\phi_{\mathrm{e}}$ and set $\phi_{\mathrm{e}}^{*}:=\sup \phi_{\mathrm{e}}$. We study the effect of the variation of $M$. We call $\phi_{M}$ the solution to Problem (5.1)-(5.2), and similarly $\kappa_{M}, \rho_{M}$. The singularity coefficient of $\phi_{M}$ is denoted by $\lambda_{M}$. Under (H1'), the singularity coefficient of the total potential $\phi_{M}-\phi_{\mathrm{e}}$ is $\Lambda_{M}=\lambda_{M}-\lambda_{\mathrm{e}}$.

Theorem 5.1. As $M \rightarrow 0$, there holds:

$$
\kappa_{M} \sim M\left(\int_{\Omega} \exp \left(\phi_{\mathrm{e}}\right) \mathrm{d} \boldsymbol{x}\right)^{-1} \text { and } \lambda_{M} \sim \kappa_{M} \int_{\Omega} \exp \left(\phi_{\mathrm{e}}\right) P_{s} \mathrm{~d} \boldsymbol{x} .
$$

Proof. From (5.1) follows that $\kappa_{M}=M\left(\int_{\Omega} \mathrm{e}^{\phi_{\mathrm{e}}-\phi_{M}}\right)^{-1}$, therefore:

$$
M\left(\int_{\Omega} \exp \left(\phi_{\mathrm{e}}\right) \mathrm{d} \boldsymbol{x}\right)^{-1} \leq \kappa_{M} \leq M \exp \left(\left\|\phi_{M}\right\|_{L^{\infty}}\right)\left(\int_{\Omega} \exp \left(\phi_{\mathrm{e}}\right) \mathrm{d} \boldsymbol{x}\right)^{-1} .
$$

As remarked above, $\Phi_{2} \subset L^{\infty}(\Omega)$, hence:

$$
\left\|\phi_{M}\right\|_{L^{\infty}} \leq C\left\|\phi_{M}\right\|_{\Phi_{2}} \leq C\left\|\rho_{M}\right\|_{L^{2}} \leq C\left\|\rho_{M}\right\|_{L^{1}}^{1 / 2}\left\|\rho_{M}\right\|_{L^{\infty}}^{1 / 2} \leq C\left(M \kappa_{M} \mathrm{e}^{\phi_{\mathrm{e}}^{*}}\right)^{1 / 2} .
$$

By Corollary 3.1, $\kappa_{M}=2 \pi \exp \mathcal{S}(M)$ is nondecreasing with respect to $M$; thus, it tends to a limit $\kappa_{0} \geq 0$ when $M \rightarrow 0$. As a consequence, we have $\left\|\phi_{M}\right\|_{L^{\infty}} \rightarrow 0$. Plugging this into (5.4) proves the first assertion in (5.3).

Similarly, we have

$$
\lambda_{M}=\int_{\Omega} \rho_{M} P_{s} \mathrm{~d} \boldsymbol{x}=\kappa_{M} \int_{\Omega} \exp \left(\phi_{\mathrm{e}}-\phi_{M}\right) P_{s} \mathrm{~d} \boldsymbol{x} .
$$

As we have seen, $\left\|\phi_{M}\right\|_{L^{\infty}} \rightarrow 0$ as $M \rightarrow 0$, therefore $\exp \left(\phi_{\mathrm{e}}-\phi_{M}\right) \rightarrow \exp \left(\phi_{\mathrm{e}}\right)$ a.e. in $\Omega$. Since $\left|\exp \left(\phi_{\mathrm{e}}-\phi_{M}\right)\right|^{2} \leq \exp \left(2 \phi_{\mathrm{e}}\right)$, the dominated convergence theorem gives $\exp \left(\phi_{\mathrm{e}}-\phi_{M}\right) \rightarrow \exp \left(\phi_{\mathrm{e}}\right)$ in $L^{2}(\Omega)$ and we obtain

$$
\frac{\lambda_{M}}{\kappa_{M}}=\int_{\Omega} \exp \left(\phi_{\mathrm{e}}-\phi_{M}\right) P_{s} \rightarrow \int_{\Omega} \exp \left(\phi_{\mathrm{e}}\right) P_{s} \quad \text { as } M \rightarrow 0,
$$

proving the second assertion in (5.3).

The behavior as $M \rightarrow+\infty$ can be investigated with the help of the theory of large solutions to non-linear elliptic problems exposed in Appendix A.

Theorem 5.2. As $M \rightarrow+\infty$, there holds:

$$
\frac{M}{\kappa_{M}} \rightarrow 0 \quad \text { and } \quad \frac{\lambda_{M}}{\kappa_{M}} \rightarrow 0 \quad \text { and } \quad \lambda_{M} \rightarrow+\infty .
$$

Proof. From (5.4), we deduce $\kappa_{M} \rightarrow+\infty$. Now, consider the function $u_{M}:=$ $\ln \rho_{M}-\phi_{\mathrm{e}}=\ln \kappa_{M}-\phi_{M}$. It appears as the unique solution in $H^{1}(\Omega)$ to:

$$
\Delta u_{M}=\rho_{M}=\exp \left(\phi_{\mathrm{e}}+u_{M}\right) \text { in } \Omega, \quad u_{M}=\ln \kappa_{M} \text { on } \Gamma_{1} \cup \Gamma_{2}, \quad \partial_{\nu} u_{M}=0 \text { on } \Gamma_{3} .
$$


As $\kappa_{M} \rightarrow+\infty, u_{M}$ converges monotonically toward the smallest solution to:

$$
\Delta u_{\infty}=\exp \left(\phi_{\mathrm{e}}+u_{\infty}\right):=\rho_{\infty} \text { in } \Omega, \quad u_{\infty}=+\infty \text { on } \Gamma_{1} \cup \Gamma_{2}, \quad \partial_{\nu} u_{\infty}=0 \text { on } \Gamma_{3} .
$$

Then, for any $\boldsymbol{x} \in \Omega, \phi_{M}(\boldsymbol{x})=\ln \kappa_{M}-u_{M}(\boldsymbol{x}) \rightarrow+\infty$ as $M \rightarrow+\infty$. Using the dominated convergence theorem, we deduce

$$
\begin{aligned}
& \frac{M}{\kappa_{M}}=\frac{1}{\kappa_{M}} \int_{\Omega} \rho_{M} \leq \mathrm{e}^{\phi_{\mathrm{e}}^{*}} \int_{\Omega} \mathrm{e}^{-\phi_{M}} \rightarrow 0, \\
& \frac{\lambda_{M}}{\kappa_{M}}=\frac{1}{\kappa_{M}} \int_{\Omega} \rho_{M} P_{s} \leq \mathrm{e}^{\phi_{\mathrm{e}}^{*}} \int_{\Omega} P_{s} \mathrm{e}^{-\phi_{M}} \rightarrow 0 .
\end{aligned}
$$

Furthermore, $P_{s} \rho_{M}$ converges monotonically toward $P_{s} \rho_{\infty}$ (recall that $P_{s}$ is nonnegative). By the monotone convergence theorem, we infer:

$$
\lambda_{M}=\int_{\Omega} P_{s} \rho_{M} \rightarrow \int_{\Omega} P_{s} \rho_{\infty}:=\lambda_{\infty} \in[0,+\infty] .
$$

Combining Eq. (4.10) with Proposition Appendix A.2 and Definition Appendix A.1, we see that, near the reentrant corner $s$ :

$$
P_{s} \rho_{\infty} \geq C \frac{r_{s}^{-\alpha_{s}} \sin \left(\alpha_{s} \theta_{s}\right)}{\left(r_{s} \sin \left(\alpha_{s} \theta_{s}\right)\right)^{2}}=\frac{C}{r_{s}^{\alpha_{s}+2} \sin \left(\alpha_{s} \theta_{s}\right)} \notin L^{1}\left(\Omega_{s}\right),
$$

hence the last part of (5.5).

Remark 5.1. To have more precise asymptotics of $\kappa_{M}$ and $\lambda_{M}$, one may think of boundary layer techniques. The relevant scale appears to be $\epsilon=\kappa_{M}^{-1 / 2}$. However, the limiting problem near the reentrant corner, to wit $-\Delta \phi=\mathrm{e}^{-\phi}$ in an infinite sector, with a Dirichlet boundary condition, seems to be ill-posed.

\subsection{Behaviour with respect to the applied voltage}

Now, we return to the Maxwell-Boltzmann problem (5.1)-(5.2), and we suppose that the value of the external potential on $\Gamma_{2}$ (the applied voltage) is given as $\phi_{\text {in }}=m \bar{\phi}_{\text {in }}$, where $m$ is a variable real parameter and

$$
\bar{\phi}_{\text {in }} \in H^{s}\left(\Gamma_{2}\right) \text { for some } s>\frac{1}{2}, \quad \min \bar{\phi}_{\text {in }}=1, \quad \max \bar{\phi}_{\text {in }}:=\bar{\phi}_{\text {in }}^{*}<+\infty .
$$

Of course, this supposes the existence of a non-empty Neumann boundary $\Gamma_{3}$ between $\Gamma_{1}$ and $\Gamma_{2}$. On the other hand, the neutralizing background density $n_{\mathrm{e}}$ and the total mass $M$ are independent of $m$. Global neutrality $\left(M=\int_{\Omega} n_{\mathrm{e}}\right)$ does not necessarily hold. This setting can be thought of as a toy model for the following situation: $\Gamma_{1}$ is the ground, with the reentrant corner representing a lightning conductor or a tall tree, $\Gamma_{2}$ is an electrically charged cloud, and $\Gamma_{3}$ is an artificial boundary which keeps the domain bounded. We call this the "cloud problem". 
We decompose $\phi_{\mathrm{e}}:=m H_{\mathrm{in}}+\psi_{\mathrm{e}}$, where $H_{\mathrm{in}}$ and $\psi_{\mathrm{e}}$ solve:

$$
\begin{aligned}
& \Delta H_{\text {in }}=0 \text { in } \Omega, \quad H_{\text {in }}=0 \text { on } \Gamma_{1}, \quad H_{\text {in }}=\bar{\phi}_{\text {in }} \text { on } \Gamma_{2}, \quad \partial_{\nu} H_{\text {in }}=0 \text { on } \Gamma_{3} ; \\
& -\Delta \psi_{\mathrm{e}}=n_{\mathrm{e}} \text { in } \Omega, \quad \psi_{\mathrm{e}}=0 \text { on } \Gamma_{1} \cup \Gamma_{2}, \quad \partial_{\nu} \psi_{\mathrm{e}}=0 \text { on } \Gamma_{3} .
\end{aligned}
$$

There holds: $0 \leq H_{\text {in }} \leq \bar{\phi}_{\text {in }}^{*}$, and $0 \leq \psi_{\mathrm{e}} \leq \psi_{\mathrm{e}}^{*}$ provided $n_{\mathrm{e}} \in H^{-1+\epsilon}(\Omega)$. Problem (5.1)-(5.2) reads:

$$
\begin{aligned}
& -\Delta \phi_{m}=\kappa_{m} \exp \left(m H_{\mathrm{in}}+\psi_{\mathrm{e}}-\phi_{m}\right):=\rho_{m} \text { in } \Omega, \quad \text { with: } \int_{\Omega} \rho_{m}=M, \\
& \phi_{m}=0 \text { on } \Gamma_{1} \cup \Gamma_{2}, \quad \partial_{\nu} \phi_{m}=0 \text { on } \Gamma_{3} .
\end{aligned}
$$

The singularity coefficient of $\phi_{m}$ is denoted $\lambda_{m}$. Under (H1'), the total singularity coefficient is $\Lambda_{m}=\lambda_{m}-m \eta_{\text {in }}-\eta_{\mathrm{e}}$, where $\eta_{\text {in }}$ and $\eta_{\mathrm{e}}$ are the singularity coefficients of $H_{\text {in }}$ and $\psi_{\mathrm{e}}$. We investigate the behavior of the various coefficients as $m \rightarrow+\infty$. The limit $m \rightarrow-\infty$ cannot be studied with the tools introduced in this article.

Theorem 5.3. As $m \rightarrow+\infty$, there holds:

$$
\kappa_{m} \rightarrow 0 \quad \text { and } \quad \kappa_{m} \exp \left(m \bar{\phi}_{\text {in }}^{*}\right) \rightarrow+\infty .
$$

Proof. Both claims are proved by contradiction. First, $\kappa_{m}$ is non-increasing with respect to $m$, as a consequence of Corollary 3.2. Thus, there exists $0 \leq \kappa_{\infty}=$ $\lim _{m \rightarrow+\infty} \kappa_{m}$. Consider $w_{m}:=\ln \rho_{m}-\psi_{\mathrm{e}}=\ln \kappa_{m}+m H_{\mathrm{in}}-\phi_{m}$, which solves the problem:

$$
\begin{aligned}
& \Delta w_{m}=\rho_{m}=\exp \left(\psi_{\mathrm{e}}+w_{m}\right) \text { in } \Omega, \\
& w_{m}=\ln \kappa_{m} \text { on } \Gamma_{1}, \quad w_{m}=\ln \kappa_{m}+m \bar{\phi}_{\text {in }} \text { on } \Gamma_{2}, \quad \partial_{\nu} w_{m}=0 \text { on } \Gamma_{3} .
\end{aligned}
$$

Assume $\kappa_{\infty}>0$. A simple comparison argument shows that $w_{m} \geq \underline{w}_{m}$, where:

$$
\begin{aligned}
& \underline{w}_{m}=\exp \left(\psi_{\mathrm{e}}+\underline{w}_{m}\right):=\underline{\rho}_{m} \text { in } \Omega, \\
& \underline{w}_{m}=\ln \kappa_{\infty} \text { on } \Gamma_{1}, \quad \underline{w}_{m}=\ln \kappa_{\infty}+m \bar{\phi}_{\text {in }} \text { on } \Gamma_{2}, \quad \partial_{\nu} \underline{w}_{m}=0 \text { on } \Gamma_{3} .
\end{aligned}
$$

As $m \rightarrow+\infty, \underline{w}_{m}$ converges monotonically (see Remark Appendix A.1) toward the smallest solution to:

$$
\begin{aligned}
& \Delta \underline{w}_{\infty}=\exp \left(\psi_{\mathrm{e}}+\underline{w}_{\infty}\right):=\underline{\rho}_{\infty} \text { in } \Omega, \\
& \underline{w}_{\infty}=\ln \kappa_{\infty} \text { on } \Gamma_{1}, \quad \underline{w}_{\infty}=+\infty \text { on } \Gamma_{2}, \quad \partial_{\nu} \underline{w}_{\infty}=0 \text { on } \Gamma_{3} ;
\end{aligned}
$$

and similarly $\underline{\rho}_{m} \nearrow \underline{\rho}_{\infty}$. By Proposition Appendix A.2 the latter is not integrable in a neighborhood of $\Gamma_{2}$. Applying the monotone convergence theorem, we find:

$$
M=\int_{\Omega} \rho_{m} \geq \int_{\Omega} \underline{\rho}_{m} \rightarrow \int_{\Omega} \underline{\rho}_{\infty}=+\infty,
$$

a contradiction which proves the first point in (5.8). 
To check the second point, assume the existence of a sequence $m_{n} \rightarrow+\infty$ such that

$$
\kappa_{m_{n}} \exp \left(m_{n} \bar{\phi}_{\mathrm{in}}^{*}\right) \leq C, \quad \text { and therefore } \kappa_{m_{n}} \exp \left(\beta m_{n}\right) \rightarrow 0, \forall \beta<\bar{\phi}_{\mathrm{in}}^{*} .
$$

As a harmonic function, $H_{\text {in }}<\bar{\phi}_{\text {in }}^{*}$ in $\Omega$. So, for all $\boldsymbol{x} \in \Omega$ one has $\rho_{m_{n}}(\boldsymbol{x}) \rightarrow 0$ while bounded by the integrable function $C \mathrm{e}^{\psi_{\mathrm{e}}}$. This implies $M=\int_{\Omega} \rho_{m_{n}} \rightarrow 0$, another contradiction.

Proposition 5.1. Assume there exists $\beta>0$ and $C>0$ such that

$$
\kappa_{m} \mathrm{e}^{\beta m} \leq C \text { as } m \rightarrow+\infty .
$$

Then the singularity coefficient $\lambda_{m}$ remains bounded as $m \rightarrow+\infty$. If (5.9) holds for all $\beta<\bar{\phi}_{\mathrm{in}}^{*}$, then $\lambda_{m} \rightarrow 0$. Therefore, $\Lambda_{m}$ is asymptotically linear in $m$.

Proof. As $H_{\text {in }}$ is continuous on $\bar{\Omega}$ and zero on $\Gamma_{1}$, the set $\omega:=\left[H_{\text {in }}<\beta\right]$ is a neighborhood of the reentrant corner $s$. On the other hand, $P_{s}$ is smooth away from $s$, so $A:=\sup _{\bar{\Omega} \backslash \omega} P_{s}$ is finite. We bound:

$$
\begin{aligned}
\lambda_{m} & =\int_{\omega} \rho_{m} P_{s}+\int_{\Omega \backslash \omega} \rho_{m} P_{s} \leq \int_{\omega} \kappa_{m} \mathrm{e}^{m H_{\mathrm{in}}} \mathrm{e}^{\psi_{\mathrm{e}}-\phi_{m}} P_{s}+A \int_{\Omega \backslash \omega} \rho_{m} \\
& \leq \int_{\omega} C \mathrm{e}^{\psi_{\mathrm{e}}^{*}} P_{s}+A M \leq C^{\prime} .
\end{aligned}
$$

If (5.9) holds for all $\beta<\bar{\phi}_{\text {in }}^{*}$, taking $\beta<\beta^{\prime}<\bar{\phi}_{\text {in }}^{*}$ shows $\kappa_{m} \mathrm{e}^{\beta m}=$ $\left(\kappa_{m} \mathrm{e}^{\beta^{\prime} m}\right) \mathrm{e}^{\left(\beta-\beta^{\prime}\right) m} \rightarrow 0$. Therefore, $\kappa_{m} \mathrm{e}^{m H_{\text {in }}(\boldsymbol{x})} \rightarrow 0$ and thus $\rho_{m}(\boldsymbol{x}) \rightarrow 0$ everywhere in $\bar{\Omega}$, except on $\Sigma:=\left[H_{\text {in }}=\bar{\phi}_{\text {in }}^{*}\right] \subset \Gamma_{2}$. As the total mass $\int_{\Omega} \rho_{m}=M$ is conserved, we infer that $\rho_{m}$ converges (in the sense of measures) toward a multiple of the surface Dirac measure $\delta_{\Sigma}$. As $P_{s}=0$ on $\Sigma \subset \Gamma_{2}$, this implies $\lambda_{m} \rightarrow 0$.

The condition (5.9) can be proved in a somewhat special geometrical setting, which nevertheless seems natural given the physical context. We assume that $\Gamma_{2}$ is a segment of a straight line, and that $\Gamma_{3}$ is made of two segments at right angles with $\Gamma_{2}$, see Figure 3 . This induces a coordinate system $(X, Y)$, with $X$ along $\Gamma_{2}$ and $Y$ along $\Gamma_{3}$. The above conditions imply that $\Omega$ is entirely contained in the half-strip $[0<Y<B$ and $X>0]$. We then choose $A^{\prime \prime}>A^{\prime}>0$ such that the rectangular domain $\Omega^{\prime}:=\left[0<X<A^{\prime}\right.$ and $\left.0<Y<B\right]$ is entirely contained in $\Omega$, which in turn is entirely contained in $\Omega^{\prime \prime}:=\left[0<X<A^{\prime \prime}\right.$ and $\left.0<Y<B\right]$. The various parts of the boundary of $\Omega^{\prime}$ will be denoted $\Gamma_{1}^{\prime}, \Gamma_{2}, \Gamma_{3}^{\prime}$, and similarly for $\Omega^{\prime \prime}$.

Proposition 5.2. Let $\Omega$ be the domain represented on Figure 3. The bound (5.9) holds for all $\beta<1$. Therefore, $\lambda_{m}$ is bounded as $m \rightarrow \infty$, and $\lambda_{m} \rightarrow 0$ if $\bar{\phi}_{\mathrm{in}}^{*}=1$.

Proof. Let $\left(m_{n}\right)_{n}$ be a sequence which tends to $+\infty$. If $\kappa_{m_{n}} \mathrm{e}^{m_{n}}$ remains bounded, then

$$
\kappa_{m_{n}} \mathrm{e}^{\beta m_{n}} \rightarrow 0 \quad \text { as } n \rightarrow+\infty, \quad \text { for all } \beta<1,
$$




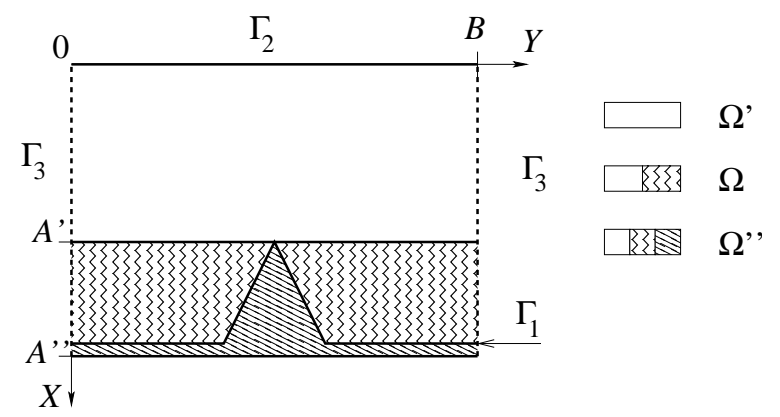

Fig. 3. Model setting for the cloud problem.

as in Proposition 5.1. To prove (5.9) for all $\beta<1$, there remains to show that (5.10) also holds for sequences such that $\kappa_{m_{n}} \mathrm{e}^{m_{n}} \rightarrow+\infty$. Let us fix one such sequence; for the sake of brevity, we write $m$ instead of $m_{n}$ from now on.

Consider the function $u_{m}:=m H_{\text {in }}-\phi_{m}=\ln \rho_{m}-\ln \kappa_{m}-\psi_{\mathrm{e}}$, which solves the problem:

$$
\begin{aligned}
& \Delta u_{m}=\rho_{m}=\kappa_{m} \exp \left(\psi_{\mathrm{e}}+u_{m}\right) \text { in } \Omega, \\
& u_{m}=0 \text { on } \Gamma_{1}, \quad u_{m}=m \bar{\phi}_{\text {in }} \text { on } \Gamma_{2}, \quad \partial_{\nu} u_{m}=0 \text { on } \Gamma_{3} .
\end{aligned}
$$

Introduce the similar function $u_{m}^{\prime}$ on $\Omega^{\prime}$, solution to:

$$
\begin{aligned}
& \Delta u_{m}^{\prime}=\rho_{m}^{\prime}:=\kappa_{m} \exp \left(\psi_{\mathrm{e}}^{*}+u_{m}^{\prime}\right) \text { in } \Omega^{\prime}, \\
& u_{m}^{\prime}=0 \text { on } \Gamma_{1}^{\prime}, \quad u_{m}^{\prime}=m \text { on } \Gamma_{2}, \quad \partial_{\nu} u_{m}^{\prime}=0 \text { on } \Gamma_{3}^{\prime},
\end{aligned}
$$

with the same value of $\kappa_{m}$. Clearly, the above problem is independent of the coordinate $Y$, and $u_{m}^{\prime}$ is given in function of $X \in\left(0, A^{\prime}\right)$ as the solution to the one-dimensional model studied in Appendix B, under the asymptotics (B.3). As noted at the end of that appendix, the one-dimensional solution can be extended to a subsolution on a larger interval, which is non-positive for $X \geq A^{\prime}$. Thus, we have extended $u_{m}^{\prime}$ to a function $\underline{u}_{m}$ on $\Omega^{\prime \prime}$, which satisfies:

$$
\begin{aligned}
& \Delta_{u} \geq \underline{\rho}_{m}:=\kappa_{m} \exp \left(\psi_{\mathrm{e}}^{*}+\underline{u}_{m}\right) \geq \kappa_{m} \exp \left(\psi_{\mathrm{e}}+\underline{u}_{m}\right) \text { in } \Omega^{\prime \prime}, \\
& \underline{u}_{m} \leq 0 \text { in } \overline{\Omega^{\prime \prime} \backslash \Omega^{\prime}}, \quad \underline{u}_{m}=m \leq m \bar{\phi}_{\text {in }} \text { on } \Gamma_{2}, \quad \partial_{\nu} \underline{u}_{m}=0 \text { on } \Gamma_{3}^{\prime \prime} .
\end{aligned}
$$

In particular, $\underline{u}_{m} \leq 0$ on $\Gamma_{1}$ : this function is a subsolution to (5.11)-(5.12). So, $\underline{u}_{m} \leq u_{m}$ in $\Omega$, which means $u_{m}^{\prime} \leq u_{m}$ in $\Omega^{\prime}$. In turn, this implies $\rho_{m}^{\prime} \leq \mathrm{e}^{\psi_{\mathrm{e}}^{*}} \rho_{m}$ and:

$$
M_{m}^{\prime}:=\int_{\Omega^{\prime}} \rho_{m}^{\prime} \leq \mathrm{e}^{\psi_{\mathrm{e}}^{*}} \int_{\Omega^{\prime}} \rho_{m} \leq \mathrm{e}^{\psi_{\mathrm{e}}^{*}} M
$$

According to Proposition Appendix B.1, $M_{m}^{\prime}$ being bounded implies $\kappa_{m} \mathrm{e}^{\beta m} \rightarrow 0$, for all $\beta<1$. 


\section{Numerical solution of the Maxwell-Boltzmann problem}

Solving numerically the problem (5.1)-(5.2) needs to tackle its non-linearity. Denoting, as in the previous sections, $\Phi[\rho]=\phi$ the solution to:

$$
-\Delta \phi=\rho \text { in } \Omega, \quad \partial_{\nu} \phi=0 \text { on } \Gamma_{3}, \quad \phi=0 \text { on } \Gamma_{1} \cup \Gamma_{2},
$$

we have used, in a finite-dimensional context which we will introduce later, the following two approaches:

(1) Simple fixed point iterations:

$$
\rho^{(k)} \stackrel{\mathcal{A}}{\longmapsto} \phi^{(k)}:=\Phi\left[\rho^{(k)}\right] \stackrel{\mathcal{B}}{\longmapsto} \rho^{(k+1)}=M \frac{\exp \left(\phi_{\mathrm{e}}-\phi^{(k)}\right)}{\int_{\Omega} \exp \left(\phi_{\mathrm{e}}-\phi^{(k)}\right)}
$$

but as the mapping $\mathcal{F}:=\mathcal{B} \circ \mathcal{A}$ is linear in $M$ it cannot be a contraction for $M$ large enough.

(2) Minimization of the functional (compare with §3.2):

$$
J[\rho]:=\int_{\Omega}\left[\rho \ln \rho-\rho-\phi_{\mathrm{e}} \rho+\frac{1}{2}|\nabla \Phi[\rho]|^{2}\right]=\int_{\Omega} \rho\left(-\phi_{\mathrm{e}}+\frac{1}{2} \Phi[\rho]+\ln \rho-1\right),
$$

on the set $L_{+}^{1}(\Omega):=\left\{v \in L^{1}(\Omega): v \geq 0\right.$ a.e. in $\Omega$ and $\left.\int_{\Omega} v=M\right\}$, which is more involved, but allows one to handle larger values of $M$.

In both approaches we need to solve many times Eq. (6.1) for various $\rho$ on a domain with a reentrant corner. It is well known that the classical $P_{1}$ finite element method generally loses the $O\left(h^{2}\right)$ convergence in $L^{2}(\Omega)$ norm. One possibility to recover the expected $O\left(h^{2}\right)$ convergence is to use the singular complement method. ${ }^{7}$ It fits well our needs, because we are also interested in studying numerically the behavior (cf. §5) of the singularity coefficient of $\phi$, which is a key ingredient of the method.

The singular complement method is used in conjunction with the classical $P_{1}$ finite element method, and we now introduce some notations we use in the sequel. Given a triangulation $\mathcal{T}^{h}$ of $\Omega$, we denote by $V^{h}$ the space spanned by the finite elements, i.e., continuous functions on $\Omega$ whose restriction on each triangle of $\mathcal{T}^{h}$ is an affine function. It is well known that each $v^{h} \in V^{h}$ can be written as:

$$
v^{h}=\sum_{i} v^{h}\left(\boldsymbol{a}_{i}\right) \varphi_{i}
$$

where the $\boldsymbol{a}_{i}$ are the triangulation vertices and $\varphi_{i}$ the global "hat" basis functions. Given a function $v^{h} \in V^{h}$, we will denote as $\left\{v^{h}\right\}$ the vector of its nodal values $\left[v^{h}\left(\boldsymbol{a}_{1}\right), \ldots, v^{h}\left(\boldsymbol{a}_{n}\right)\right]^{\top}$ and $\mathbb{M}$ the mass matrix with coefficients $\mathbb{M}_{i, j}=\left(\varphi_{i}, \varphi_{j}\right)$. Using the mass matrix, the $L^{2}$ scalar product between two functions of $V^{h}$ can be computed by $\left(u^{h}, v^{h}\right)=\left\{u^{h}\right\}^{\top} \mathbb{M}\left\{v^{h}\right\}$. Moreover we will denote:

- $\Pi_{I}^{h}$ the interpolation operator from $C(\bar{\Omega})$ to $V^{h}: \Pi_{I}^{h} v=\sum_{i} v\left(\boldsymbol{a}_{i}\right) \varphi_{i}$;

- $\Pi^{h}$ the orthogonal projection operator from $L^{2}(\Omega)$ to $V^{h}$; for any $v \in L^{2}(\Omega)$ we have $\Pi^{h} v=\sum_{i} w_{i} \varphi_{i}$ with $w \in \mathbb{R}^{n}$ the solution of the linear system $\mathbb{M} w=b$, with $b_{i}=\left(v, \varphi_{i}\right)$. 
The subspace $V_{0}^{h} \subset V^{h}$ of functions which vanish on $\Gamma_{1} \cup \Gamma_{2}$ is used to approximate the regular part of $\Phi[\rho]$ (cf. $\S 4$ ), while its singular part is treated in a semi-analytical way which we detail below. A similar approach is used for $\phi_{\mathrm{e}}$. Finally we also use the same space $V^{h}$ to approximate the function $\rho$ in both fixed point and minimisation approaches. This is not mandatory, but overall simplifies the computations.

The rest of this section is organized as follows. First we recall briefly the principles of the singular complement method. Then we give some details on our two approaches to solve the non-linear problem. Finally we present numerical tests on the two family of problems examined in $\S 5$, the behavior with respect to the mass and the behavior with respect to the applied voltage (the cloud problem), and compare the results with the expected theoretical behavior.

\subsection{The singular complement method}

The method is based on the following decomposition ${ }^{12}$ of $L^{2}(\Omega)$ :

$L^{2}(\Omega)=\Delta \Phi_{2} \stackrel{\perp}{\oplus} N, \quad$ where: $\quad N=\left\{p \in L^{2}(\Omega): \Delta p=0, p_{\mid \Gamma_{1} \cup \Gamma_{2}}=0, \partial_{\nu} p_{\mid \Gamma_{3}}=0\right\}$, see Definition 3.3 for $\Phi_{2}$. In case of only one reentrant corner $\operatorname{dim} N=1$ and $N=\operatorname{span}\left(P_{s}\right)$, see (4.7)-(4.8). So we split the right hand side of (6.1) as:

$$
\rho:=\rho_{R}+\rho_{S}, \quad \text { where: } \quad \rho_{S}=\frac{\left(\rho, P_{s}\right)}{\left\|P_{s}\right\|_{L^{2}}^{2}} P_{s}:=c P_{s}, \quad \rho_{R}=\rho-\rho_{S},
$$

then by linearity $\Phi[\rho]=\Phi\left[\rho_{R}\right]+\Phi\left[\rho_{S}\right]=\Phi\left[\rho_{R}\right]+c \Phi\left[P_{s}\right]$. This decomposition is equivalent to (4.3).

In the following we denote $\phi_{s}:=\Phi\left[P_{s}\right], \varkappa=\left\|P_{s}\right\|_{L^{2}}^{2}, \lambda=\left(\rho, P_{s}\right)$ the singularity coefficient. If the domain $\Omega$ and the boundary conditions are fixed, one can compute once and for all the dual and primal singular functions $P_{s}$ and $\phi_{s}$, and the coefficient $\varkappa$. Practically, we compute approximations $P_{s}^{h}, \phi_{s}^{h}, \varkappa^{h}$ on the given mesh $\mathcal{T}^{h}$, which should respect a few constraints on the triangles around the reentrant corner. This must be done with care, see Appendix C for details. Summarizing, a function $\rho$ being given, the method consists in:

(1) computing the singularity coefficient $\lambda^{h}=\left(\rho, P_{s}^{h}\right)$; for $\rho \in V^{h}$ this reads $\lambda^{h}=$ $L^{\top}\{\rho\}$ with $L_{i}=\left(\varphi_{i}, P_{s}^{h}\right)$

(2) approximating $\phi_{R}:=\Phi\left[\rho_{R}\right]$ by $\phi_{R}^{h}$, using the finite element method;

(3) finally, reconstituting $\phi^{h}:=\phi_{R}^{h}+c^{h} \phi_{s}^{h}$, with $c^{h}=\lambda^{h} / \varkappa^{h}$.

\subsection{The nonlinear solving procedures}

Fixed point iterations

In our finite-dimensional context, the operator $\mathcal{A}^{h}$ corresponding to $\mathcal{A}$ is the one obtained by the method described just above. It goes from $V^{h}$ to $V_{0}^{h}$. Now, it is clear that the operator $\mathcal{B}$ does not map $V_{0}^{h}$ to $V^{h}$. Therefore, we tried two possible discrete 
versions, namely (i) $\mathcal{B}^{h}=\Pi_{I}^{h} \circ \mathcal{B}$ and (ii) $\mathcal{B}^{h}=\Pi^{h} \circ \mathcal{B}$. The second approach is more time-consuming (as it involves the solution of a linear system with matrix $\mathbb{M}$ ), but it causes the fixed point iterations to converge until a slightly larger value of $M$ than the first one. As stopping criterion we chose:

$$
\left\|\rho^{(k)}-\rho^{(k+1)}\right\|_{L^{2}}^{2} \leq t o l\left\|\rho^{(k+1)}\right\|_{L^{2}}^{2}
$$

with $t o l=10^{-8}$.

\section{Optimization procedure}

Denoting $\tilde{J}(\{\rho\}):=J[\rho]$ we have a minimization problem in $\mathbb{R}^{n}$ which can be solved by an appropriate method. We used the limited memory quasi-Newton m1qn3 code. ${ }^{10}$ As the operator $\rho \mapsto \Phi[\rho]$ is self-adjoint, we get easily:

$$
\frac{\partial \tilde{J}}{\partial\{\rho\}_{i}}(\{\rho\})=\left\langle J^{\prime}[\rho], \varphi_{i}\right\rangle=\int_{\Omega} \varphi_{i}\left(-\phi_{\mathrm{e}}+\Phi[\rho]+\ln \rho\right)
$$

m1qn3 does not handle constraints, but starting from $\rho^{(0)} \in V^{h}$ which verifies the mass constraint and projecting the gradient onto the subspace of $V^{h}$ made of functions with vanishing integral, the whole optimization process takes place in $\left\{\rho \in V^{h}: \int_{\Omega} \rho=M\right\}$. A key point is to use the $L^{2}(\Omega)$ scalar product, which corresponds to the $\mathbb{R}^{n}$ scalar product $u^{\top} \mathbb{M} v$. This can be done easily by a Cholesky factorization of $\mathbb{M}$. Indeed, even on quite regular meshes, we remarked a good improvement when using this scalar product instead of the canonical one.

Despite the a priori good properties of the functional $J$ in view of minimization, we guess it is quite flat near the optimum. So to have a better idea of the solution quality, we do one fixed point iteration at the end of the optimization. Namely, from $\rho$ obtained by minimization, we compute $\rho^{r}=\mathcal{B}^{h} \circ \mathcal{A}^{h} \rho$ and measure the relative variation $\left\|\rho-\rho^{r}\right\|_{L^{2}}^{2} /\left\|\rho^{r}\right\|_{L^{2}}^{2}$. Then, $\rho^{r}$ is chosen as a new starting point for the minimization. Repeating this a few times, we select the iterate with the smallest relative variation. As we use the optimization procedure when $M$ is large enough (so that $\mathcal{B}^{h} \circ \mathcal{A}^{h}$ is not contracting), the underlying idea is to "just try another starting point" not too far from the optimum.

\subsection{Some numerical tests}

Behaviour with respect to the mass $M$

For these tests we take $\phi_{\mathrm{e}}=0$, and a Dirichlet boundary condition everywhere for $\phi$, i.e., $\Gamma_{1}=\partial \Omega$. We go from small values of $M(0.01)$ up to the largest one $(M=1000)$ we could manage with a good reliability of the results, i.e., with $\left\|\rho-\rho^{r}\right\|_{L^{2}}^{2} /\left\|\rho^{r}\right\|_{L^{2}}^{2} \leq 0.5 \times 10^{-3}$. An example of computation $(M=400)$ is plotted on Figure 4.

Figure 5 shows: 

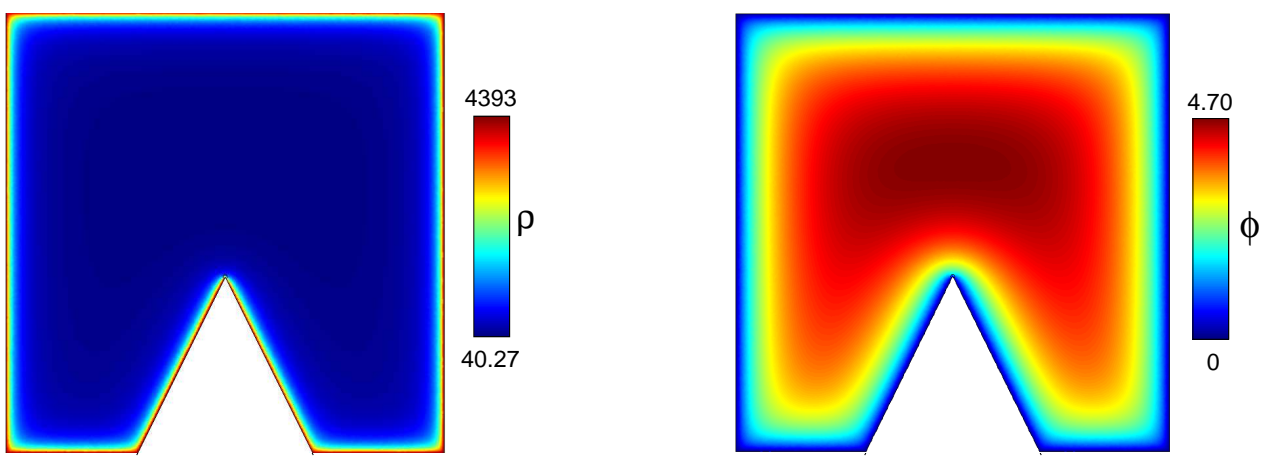

Fig. 4. The solution for $M=400$. Left: density $\rho$; right: potential $\phi$.

(1) $\lambda$ as function of $M$ : we note a nearly linear dependency, but the curve has a slight concavity (the dotted line is the straight line between the two extreme points).

(2) $M / \kappa$ as function of $M$ in $\log -\log$ scale: for $M \leq 1$, the least-squares line gives a slope of -0.0047 which is consistent with Theorem 5.1. Moreover, one checks the asymptotics $M / \kappa \widetilde{0}\left(\int_{\Omega} \exp \left(\phi_{\mathrm{e}}\right) \mathrm{d} \boldsymbol{x}\right)^{-1}=|\Omega|^{-1}$.

For large $M$, the results agree with Theorem 5.2 ; the least-squares line (computed for $M \geq 200$ ) have a slope around -0.9 (so that $\kappa \simeq C M^{1.9}$ for $M \in[200,1000])$ which seems to show that $\kappa$ would be a sub-quadratic function of $M$ when $M \rightarrow \infty$.

(3) $\lambda / \kappa$ as function of $M$ in log-log scale: as previously, the numerical results are well consistent with both Theorems 5.1 and 5.2. For $M \geq 200$ the least-squares line has a slope of -0.955 which implies that $\lambda$ would be a slightly sub-linear function of $M$ when $M \rightarrow \infty$, which is in accordance with the slight concavity of the $\lambda_{M}$ curve.

Remark 6.1. The switch from fixed point to optimization procedures occurs at $M=85$. As $M$ becomes large, the density $\rho$ concentrates near the boundary: a thin boundary layer appears (see Figure 4), which needs a very fine mesh at least near the boundary. Thus one faces a dilemma. Using an adapted mesh typically causes the condition number of the mass and stiffness matrices to grow fast, and may amplify errors in our stopping criterion. On the other hand, a very regular mesh needs a high number of nodes. This limited to $M \leq 1000$ the range of tractable problems on our computer.

Behavior with respect to the applied voltage (the cloud problem)

In this test, we choose $\phi_{\mathrm{e}}$ to be the solution of $(2.9)-(2.10)$, where $\phi_{\text {in }}=m \in \mathbb{R}$ is constant. Hence, the assumptions of Proposition 5.2 hold, as the domain $\Omega$ is of the type depicted on Figure 3 (cf. Figure 8 in Appendix C); and $\bar{\phi}_{\text {in }} \equiv 1$ is constant. 

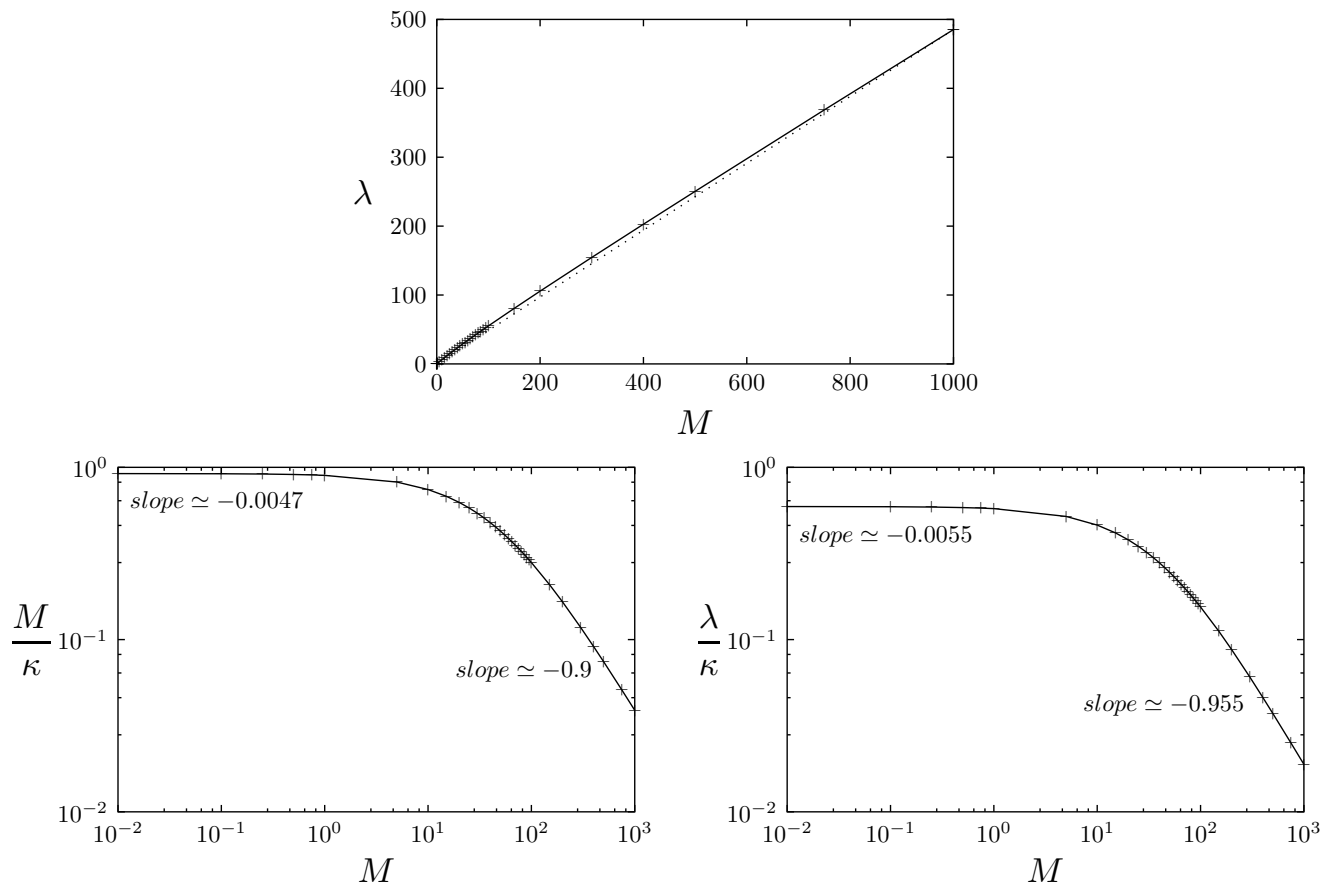

Fig. 5. Behavior with respect to the mass

Furthermore, $n_{\mathrm{e}} \equiv 10$ is also constant, and the mass is fixed to $M=n_{\mathrm{e}}|\Omega|$, which is small enough to use the fixed point method. Each numerical test corresponds to an $m$ (voltage parameter) picked in the interval $[-200,400] . \phi_{\mathrm{e}}$ is computed the same way as $\Phi[\rho]$, up to a lifting of the non-homogeneous Dirichlet condition on $\Gamma_{2}$. Our numerical results are displayed on Figure 6, and seem to be consistent with Theorem 5.3 and Proposition 5.1:

- The curve $\kappa$ as a function of $m$ in y-log scale: the least-squares line (computed with the 5 points with $m \geq 100$ ) has a slope of -0.9955 (the last segment has a slope of -0.9971$)$ so that ultimately it seems that the limiting slope would be almost $-1\left(\kappa \leq C e^{-\beta m}\right.$ for all $\beta<1$, when $\left.m \rightarrow \infty\right)$. This is in agreement with Proposition 5.1, as $\bar{\phi}_{\text {in }}^{*}=1$.

- The curve $\lambda$ as a function of $m$ : clearly $\lambda$ seems to go to 0 as $m \rightarrow+\infty$. The same curve in y-log scale exhibits a slightly convex (rather than straight) appearance for $m$ large, hinting at a sub-exponential decay of $\lambda$.

- As $m \rightarrow-\infty, \kappa$ seems to grow in a sub-exponential fashion, while $\lambda \rightarrow 0$ again.

Remark 6.2. As $m$ tends to $+\infty$ (resp. $-\infty$ ), a very thin boundary layer appears at the top (resp. the bottom) of the domain. To capture it, the mesh is strongly refined in this region. Using such a graded mesh is computationally not too difficult, 
as we use the fixed point method.
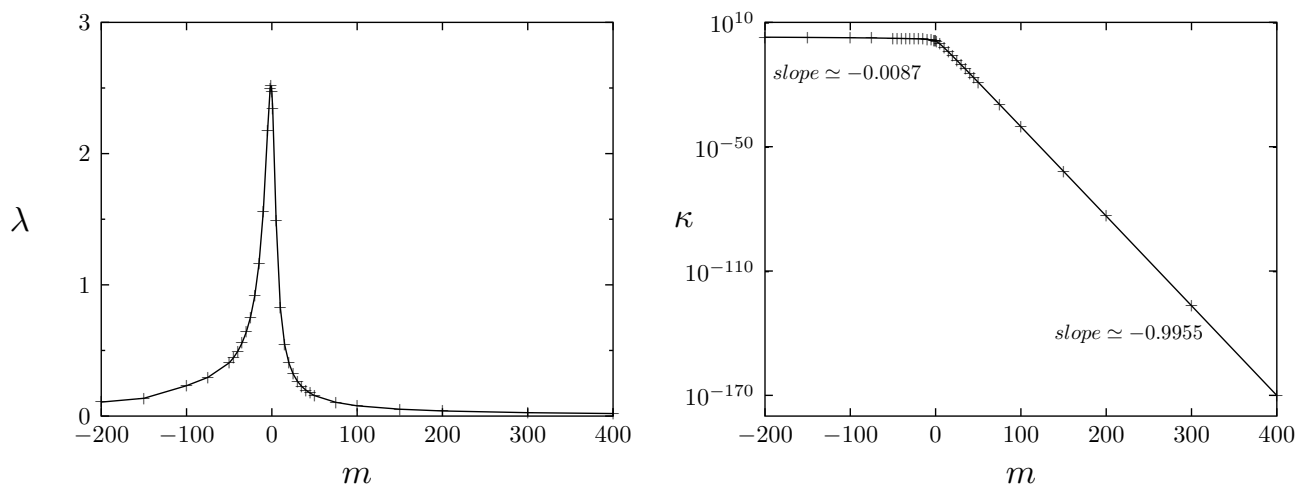

Fig. 6. Results for the cloud problem

\section{Appendix A. Large solutions to $\Delta u=p \mathrm{e}^{u}$ in a polygon}

We consider the problem, set in a bounded Lipschitz domain $\Omega$ :

$$
\begin{aligned}
& \Delta u=p(\boldsymbol{x}) \mathrm{e}^{u} \text { in } \Omega, \quad \text { with } p \geq 0 \text { a.e. in } \Omega ; \\
& u \rightarrow+\infty \text { near } \Gamma_{B}, \quad u=g_{D} \text { on } \Gamma_{D}, \quad \partial_{\nu} u=g_{N} \text { on } \Gamma_{N} .
\end{aligned}
$$

The first part of (A.2) will be called a Bieberbach condition. ${ }^{3}$ The Dirichlet and Neumann parts $\Gamma_{D}$ and $\Gamma_{N}$ of the boundary can be empty. There is, however, a restriction: if $\Gamma_{D}$ is not empty, then $\Gamma_{N} \neq \emptyset$ and $\bar{\Gamma}_{B} \cap \bar{\Gamma}_{D}=\emptyset$, i.e., a Dirichlet part and a Bieberbach part are always separated by a Neumann part.

The pure Bieberbach problem $\left(\Gamma_{B}=\partial \Omega\right)$ has been intensively studied, see Ref. 18 and references therein. However, most results were proved in the framework of smooth domains, and to our knowledge mixed conditions were not considered. Here, we prove the existence and (under some restrictive conditions) uniqueness of the solution to (A.1)-(A.2), and an asymptotic expansion in the neighborhood of $\Gamma_{B}$. We follow the approach of Ref. 9, to which we refer for the omitted details. We shall concentrate on the novelties induced by the presence of corners and the (possibly) mixed boundary conditions.

Existence. Let us begin with a special case. If $\Omega$ is the disk $B\left(\boldsymbol{x}_{0}, R\right)$, the function

$$
u_{\mathrm{d}}(\boldsymbol{x}):=-2 \ln \left(\frac{R^{2}-\left|\boldsymbol{x}-\boldsymbol{x}_{0}\right|^{2}}{2 R} \sqrt{\frac{p_{*}}{2}}\right)
$$

is solution to (A.1)-(A.2) for $p=p_{*}=$ constant and $\Gamma_{B}=\partial \Omega$. (According to Ref. 18 , it is the only one.) It is also solution to the mixed Bieberbach-Neumann 
problem (with $g_{N}=0$ ) if $\Omega$ is a sector of the previous disk with vertex at $\boldsymbol{x}_{0}$, $\Gamma_{B}$ is the $\operatorname{arc}\left[\left|\boldsymbol{x}-\boldsymbol{x}_{0}\right|=R\right]$, and $\Gamma_{N}$ is the rest of the boundary, i.e., the two radii converging to $\boldsymbol{x}_{0}$.

Now we return to the case where $\Omega$ is a polygon.

Proposition Appendix A.1. Assume that $0<p_{*} \leq p(\boldsymbol{x}) \leq p^{*}<+\infty, g_{D} \in$ $H^{1 / 2+\epsilon}\left(\Gamma_{D}\right)$ and $g_{N} \in H^{-1 / 2+\epsilon}\left(\Gamma_{N}\right)$ for some $\epsilon>0$. There exists at least one solution $u$ to (A.1)-(A.2).

Proof. Assume for the moment that $g_{N}=0$ or $g_{D}=0$, whenever $\Gamma_{N}$ or $\Gamma_{D}$ is not empty. From a variational argument (cf. Proposition 3.3), there exists a unique solution $u_{K} \in H^{1}(\Omega)$ to

$$
\Delta u_{K}=p(\boldsymbol{x}) \mathrm{e}^{u_{K}} \text { in } \Omega, \quad u_{K}=K \text { on } \Gamma_{B}, \quad u_{K}=0 \text { on } \Gamma_{D}, \quad \partial_{\nu} u_{K}=0 \text { on } \Gamma_{N},
$$

and it belongs to $C(\bar{\Omega})$, see $\S 4$. The monotonicity of the exponential implies $u_{K_{1}} \geq$ $u_{K_{2}}$ on $\bar{\Omega}$ for $K_{1} \geq K_{2} \cdot{ }^{19}$ Thus, for any $\boldsymbol{x} \in \bar{\Omega}$, there exists a (finite or not) limit $u(\boldsymbol{x})=\lim _{K \rightarrow+\infty} u_{K}(\boldsymbol{x})$. Clearly, $u$ is zero on $\Gamma_{D}$ and infinite on $\Gamma_{B}$. Moreover, for any $A>0$ and $K>A$ there is a neighborhood $\mathcal{U}$ of $\Gamma_{B}$ such that $u_{K}>A$ in $\mathcal{U}$, and hence $u>A$ in $\mathcal{U}$ : this is the Bieberbach condition.

Using again a comparison argument, we see that if $\boldsymbol{x}_{0} \in \Omega$ and $R$ is small enough to have $\omega=B\left(\boldsymbol{x}_{0}, R\right) \subset \Omega$, then for any $K, u_{K}$ is majorized in $\omega$ by $u_{\mathrm{d}}$ defined in (A.3). The same holds on the sector or half-disk $\omega=B\left(\boldsymbol{x}_{0}, R\right) \cap \Omega$, with either $\boldsymbol{x}_{0} \in \Gamma_{N}$ or $\boldsymbol{x}_{0} \in \bar{\Gamma}_{D}$ and $R$ small enough. Thus in all three cases $u \leq u_{\mathrm{d}}$ in $\omega$, and $u$ is bounded on a slightly smaller neighborhood. By a compactness argument, $u \in L^{\infty}(\omega)$ for any $\omega \subset \Omega$ such that $\bar{\omega} \cap \Gamma_{B}=\emptyset$. Similarly, $p(\boldsymbol{x}) \exp u_{K}(\boldsymbol{x}) \rightarrow$ $p(\boldsymbol{x}) \exp u(\boldsymbol{x})$ monotonically for all $\boldsymbol{x} \in \bar{\Omega}$. By the monotone convergence theorem, this implies $u_{K} \rightarrow u$ and $p \mathrm{e}^{u_{K}} \rightarrow p \mathrm{e}^{u}$ strongly in $L^{2}(\omega)$. Therefore, $\Delta u=p \mathrm{e}^{u}$ in the sense of distributions.

Let $\omega$ be a polygonal subdomain equal to $\Omega$ minus some neighborhood of $\Gamma_{B}$. As $u \in L^{2}(\omega)$ and $\Delta u \in L^{2}(\omega)$, one can consider ${ }^{12}$ the traces of $u$ and $\partial_{\nu} u$ in a very weak sense on each side of $\partial \omega$; by continuity, $\partial_{\nu} u=0$ on $\partial \omega \cap \Gamma_{N}$. Together with $u=0$ on $\Gamma_{D} \cap \partial \omega$ and $u \in L^{\infty}(\omega)$, this fact implies that $u$ is actually of $H^{1}$ regularity, except maybe near the remaining part of $\partial \omega \cdot{ }^{12}$ Thus, the Neumann condition holds in the sense of $H^{-1 / 2}\left(\Gamma_{N}^{\prime}\right)$, for any $\Gamma_{N}^{\prime} \subset \Gamma_{N}$ such that $\bar{\Gamma}_{N}^{\prime} \cap \bar{\Gamma}_{B}=\emptyset$.

To prove the general case, introduce the function $v \in H^{1}(\Omega)$ solution to

$$
\Delta v=0 \text { in } \Omega, \quad v=0 \text { on } \Gamma_{B}, \quad v=g_{D} \text { on } \Gamma_{D}, \quad \partial_{\nu} v=g_{N} \text { on } \Gamma_{N} .
$$

The conditions on the boundary data imply that $v \in H^{1+\epsilon}(\Omega) \subset C(\bar{\Omega})$ for $\epsilon$ small enough. Therefore, $p_{\circ}:=p \mathrm{e}^{v}$ is bounded above and below like $p$. We have just seen that there exists $u_{\circ}$ such that

$$
\Delta u_{\circ}=p_{\circ}(\boldsymbol{x}) \mathrm{e}^{u_{\circ}} \text { in } \Omega, \quad u_{\circ}=+\infty \text { on } \Gamma_{B}, \quad u_{\circ}=0 \text { on } \Gamma_{D}, \quad \partial_{\nu} u_{\circ}=0 \text { on } \Gamma_{N} .
$$


Finally, $u:=u_{\circ}+v$ is solution to (A.1)-(A.2).

Remark Appendix A.1. Setting aside the question of uniqueness, the above procedure necessarily yields the smallest solution to (A.1)-(A.2), which is by definition unique. For instance, taking the limit of $u_{K}$ such that:

$\Delta u_{K}=p(\boldsymbol{x}) \mathrm{e}^{u_{K}}$ in $\Omega, \quad u_{K}=\varphi_{K}$ on $\Gamma_{B}, \quad u_{K}=g_{D}$ on $\Gamma_{D}, \quad \partial_{\nu} u_{K}=g_{N}$ on $\Gamma_{N}$, with $\varphi_{K}(\boldsymbol{x}) \nearrow+\infty$ uniformly in $\boldsymbol{x}$ as $K \rightarrow+\infty$, one finds the same solution.

Remark Appendix A.2. Let $p^{1} \leq p^{2}$ be as in Proposition Appendix A.1, and $u^{1}, u^{2}$ be the corresponding smallest solutions to (A.1)-(A.2). Comparing the approximating sequences $u_{K}^{1}, u_{K}^{2}$, one finds $u^{1} \geq u^{2}$.

Expansion near the Bieberbach boundary and uniqueness. For the moment, we suppose that

$$
p=\text { const. in } \Omega \text { and } g_{N} \equiv 0 \text { on } \Gamma_{N} \cap \partial \mathcal{V},
$$

where $\mathcal{V}$ is a neighborhood of $\Gamma_{B}$ in $\Omega$. To describe the behavior of $u$ in $\mathcal{V}$, we introduce the following functions. For any side $\Gamma_{i} \subset \Gamma_{B}$ and $\boldsymbol{x} \in \Omega$, let $d_{i}(\boldsymbol{x})$ be the distance to $\Gamma_{i}$. In a neighborhood of $\Gamma_{i}$, there holds $-\nabla d_{i}=\boldsymbol{\nu}_{i}$, the normal vector, and $\Delta d_{i}=0$. Now, consider a pure Bieberbach corner $c \in \Gamma_{B}$, of opening $\pi / \alpha_{c}$. Let $\left(r_{c}, \theta_{c}\right)$ be the local polar coordinates as in $\S 4$. We define:

$$
d_{c}(\boldsymbol{x})=d_{c}\left(r_{c}, \theta_{c}\right):=\alpha_{c}^{-1} r_{c} \sin \left(\alpha_{c} \theta_{c}\right) .
$$

For a mixed Bieberbach-Neumann corner $c \in \bar{\Gamma}_{B} \cap \bar{\Gamma}_{N}$, we take again the definition (A.5), but $\alpha_{c}$ is now such that the opening is $\pi /\left(2 \alpha_{c}\right)$, and $\theta_{c}=0$ is the Bieberbach side. In both cases, simple calculations show that $-\nabla d_{c}= \pm \mathbf{e}_{\theta_{c}}=\boldsymbol{\nu}$ on the Bieberbach side(s), and $\nabla d_{c}=\mathbf{e}_{r_{c}}$ on the Neumann side of a mixed corner, i.e., $\partial_{\nu} d_{c}=0$. Furthermore:

$$
\min \left(\alpha_{c}^{2}, \alpha_{c}^{-2}\right) \leq\left|\nabla d_{c}\right|^{2} \leq \max \left(\alpha_{c}^{2}, \alpha_{c}^{-2}\right) \quad \text { and } \quad\left|\nabla d_{c}\right|^{2}-d_{c} \Delta d_{c}=1 .
$$

Using a partition of unity in $\mathcal{V}$, we construct the following function.

Definition Appendix A.1. The pseudo-distance $d$ to $\Gamma_{B}$ is a function which is equal to $d_{i}$ near the midpoint of each side $\Gamma_{i} \subset \Gamma_{B}$, to $d_{c}$ near each corner $c \in \bar{\Gamma}_{B}$ (including the mixed corners), and varies smoothly in between. These respective regions will be called the mid-edge zone, the corner zone and the transition zone. There holds: $d(\boldsymbol{x})=0 \Longleftrightarrow \boldsymbol{x} \in \Gamma_{B}$, and $d$ is uniformly bounded above and below by the actual distance to $\Gamma_{B}$.

If $\mathcal{V}$ is not too large, then $d \in C^{2}(\mathcal{V})$, and this regularity extends all the way to $\Gamma_{B}$, except the corners. In the transition zone, the property $-\Delta d=\boldsymbol{\nu}$ on $\Gamma_{B}$ is still verified; so there exist four constants such that:

$$
0<C_{1} \leq|\nabla d|^{2} \leq C_{2},\left.\quad|1-| \nabla d\right|^{2}\left|\leq C_{3} d, \quad\right| \Delta d \mid \leq C_{4} .
$$

Recall that $C_{1}=C_{2}=1$ and $C_{3}=C_{4}=0$ in the mid-edge zone. As $\nabla d \neq 0$ everywhere, the level sets $[d=\delta]$ are $C^{2}$ submanifolds for all $\delta>0$. 
Theorem Appendix A.1. Under (A.4), the solution to (A.1)-(A.2) is unique, and satisfies:

$$
\lim _{\boldsymbol{x} \rightarrow \Gamma_{B}} u(\boldsymbol{x})-\ln \left[\frac{2}{p d(\boldsymbol{x})^{2}}\right]=0
$$

Proof. Thanks to the regularity properties of $d$, one can follow the lines of Ref. 9, and prove that any solution $u$ satisfies

$$
\lim _{\boldsymbol{x} \rightarrow \Gamma_{B}} \frac{u(\boldsymbol{x})}{-2 \ln d(\boldsymbol{x})}=1
$$

Therefore, any two solutions are equivalent near $\Gamma_{B}$, which allows one to prove uniqueness.

To obtain the stronger estimate (A.8), we construct suitable super- and subsolutions in the neighborhood $\Omega_{\delta}:=[d<\delta]$. Introduce the function

$$
\bar{u}:=-2 \ln d+\ln \left(\frac{2+\epsilon}{p}\right)+K d^{\beta},
$$

where $\epsilon>0, K>0$ and $0<\beta<1$ are constants. One readily checks that:

$$
\begin{aligned}
\Delta \bar{u} & =d^{-2}\left[2\left(|\nabla d|^{2}-d \Delta d\right)+K \beta\left[(\beta-1)|\nabla d|^{2}+d \Delta d\right] d^{\beta}\right] ; \\
p \mathrm{e}^{\bar{u}} & =(2+\epsilon) d^{-2} \exp \left(K d^{\beta}\right) .
\end{aligned}
$$

In the corner zone, Eq. (A.6) shows that

$$
\Delta \bar{u}=d^{-2}\left[2+K \beta\left(\beta|\nabla d|^{2}-1\right) d^{\beta}\right] ;
$$

this implies $\Delta \bar{u} \leq p \mathrm{e}^{\bar{u}}$ when $0<\beta<\min \left(\alpha_{c}^{2}, \alpha_{c}^{-2}\right)$, for any $K$ and $\epsilon$. In the mid-edge and transition zones, the bounds (A.7) imply:

$$
\Delta \bar{u} \leq d^{-2}\left[2+2\left(C_{3}+C_{4}\right) d+K \beta\left[(\beta-1) C_{1}+C_{3} d\right] d^{\beta}\right] .
$$

For any $\beta<1$ and $\epsilon>0$, there holds $\Delta \bar{u} \leq p \mathrm{e}^{\bar{u}}$ in $\Omega_{\delta}$, regardless the size of $K$, if $\delta$ is small enough. Similarly, it can be checked that the function

$$
\underline{u}:=-2 \ln d+\ln \left(\frac{2-\epsilon}{p}\right)-K d^{\beta},
$$

satisfies $\Delta \underline{u} \leq p \mathrm{e}^{\underline{u}}$ in $\Omega_{\delta}$, if $\beta<\min \left(\alpha_{c}^{2}, \alpha_{c}^{-2}\right)$ and $\delta$ is small enough.

If $K$ is large enough, one can achieve $\underline{u} \leq u \leq \bar{u}$ on the $[d=\delta]$ part of the boundary; while on the remaining part $\partial \Omega_{\delta} \cap \Gamma_{N}$ there holds $\partial_{\nu} \underline{u}=\partial_{\nu} \bar{u}=0$. Therefore, $\bar{u}$ and $\underline{u}$ are respectively a supersolution and a subsolution in $\Omega_{\delta}$; as $u$ is the unique solution, this implies $\underline{u}(\boldsymbol{x}) \leq u(\boldsymbol{x}) \leq \bar{u}(\boldsymbol{x})$ in $\Omega_{\delta}$, for any $\epsilon>0$. Taking the limits $\epsilon \rightarrow 0$ and $d(\boldsymbol{x}) \rightarrow 0$ yields (A.8).

As an immediate consequence of (A.8), one has:

$$
p \mathrm{e}^{u(\boldsymbol{x})} \sim 2 d(\boldsymbol{x})^{-2} \text { as } \boldsymbol{x} \rightarrow \Gamma_{B} .
$$


The question of uniqueness in the general case is still open. However, one can derive useful estimates for the smallest solution to (A.1)-(A.2) when $p$ is variable, but $g_{N}$ still vanishes near $\Gamma_{B}$. To this end, introduce the (unique) solutions $u_{*}$ and $u^{*}$ obtained by replacing $p$ with $p_{*}$ and $p^{*}$ in (A.1). By Remark Appendix A.2, $u^{*} \leq u \leq u_{*}$, hence:

$$
\liminf _{\boldsymbol{x} \rightarrow \Gamma_{B}} u(\boldsymbol{x})-\ln \left[\frac{2}{p^{*} d(\boldsymbol{x})^{2}}\right] \geq 0 \quad \text { and } \quad \limsup _{\boldsymbol{x} \rightarrow \Gamma_{B}} u(\boldsymbol{x})-\ln \left[\frac{2}{p_{*} d(\boldsymbol{x})^{2}}\right] \leq 0 .
$$

Thus we are led to:

Proposition Appendix A.2. If $g_{N}$ vanishes near $\Gamma_{B}$, the smallest solution to (A.1)-(A.2) satisfies

$$
C_{1} d(\boldsymbol{x})^{-2} \leq p(\boldsymbol{x}) \mathrm{e}^{u(\boldsymbol{x})} \leq C_{2} d(\boldsymbol{x})^{-2},
$$

for some constants $C_{1}<C_{2}$, if $d(\boldsymbol{x})$ is small enough.

\section{Appendix B. One-dimensional model for the cloud problem}

In this appendix we are interested in the following problem:

$$
\begin{aligned}
& u^{\prime \prime}(x)=p \mathrm{e}^{u(x)} \text { in }(0, L), \quad \text { with } p>0 \text { constant } \\
& u(0)=m>0, \quad u(L)=0 .
\end{aligned}
$$

The interval length $L$ is fixed, and we investigate the asymptotic behavior as

$$
p \rightarrow 0 \text { and } m \rightarrow+\infty \text { while } p \mathrm{e}^{m} \rightarrow+\infty \text {. }
$$

Accordingly, we shall use to word "eventually" as a shorthand meaning "for $p$ small enough, and/or $m$ large enough and/or $p \mathrm{e}^{m}$ large enough".

The solution $u$ is of course unique but its analytical expression differs according to the values of the parameters $L, p, m$. If $L<\left(1-\mathrm{e}^{-m / 2}\right) \sqrt{2 / p}-$ which is eventually true - it is given as:

$$
u(x)=-2 \ln \sinh (a(x-L)+b)+\ln \left(2 a^{2} / p\right)
$$

where the parameters $a>0, b$ are given by the conditions (B.2), which read:

$$
\sinh b=a \sqrt{\frac{2}{p}} \text { and } \sinh (b-a L)=a \sqrt{\frac{2}{p \mathrm{e}^{m}}} .
$$

Eliminating $b$, we find the following implicit equation for $a$ :

$$
a \sqrt{\frac{2}{p \mathrm{e}^{m}}}=\sinh \left[\operatorname{arcsinh}\left(a \sqrt{\frac{2}{p}}\right)-L a\right]:=f(a) .
$$


Asymptotic behavior of $\boldsymbol{a}$. The function $f(s)$ is increasing for $s$ small, maximal for $s=s^{*}:=\sqrt{L^{-2}-p / 2}$, and tends to $-\infty$ as $s \rightarrow+\infty$, as shown on Figure 7 . Furthermore, it is concave at least for $s \leq s^{*}$. Under (B.3), $f\left(s^{*}\right) \rightarrow+\infty$, while $s^{*} \leq L^{-1}$. On the other hand, $s^{*} \sqrt{2 / p \mathrm{e}^{m}} \rightarrow 0$. We infer that, eventually, the equality (B.6) can only happen in the descending part of the curve of $f$, i.e., for $a>s^{*}$; this ensures the uniqueness of $a$. Then, the conditions (B.5) imply $b \rightarrow+\infty$ and $b-a L \rightarrow 0$, hence $a \rightarrow+\infty$ under (B.3).

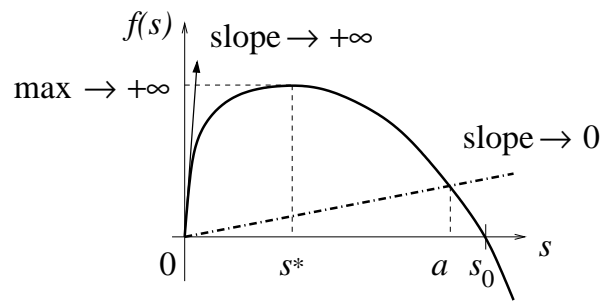

Fig. 7. Solution of Eq. (B.6).

On the other hand, there holds $a<s_{0}$, where $f\left(s_{0}\right)=0$, i.e.:

$$
s_{0} \sqrt{\frac{2}{p}}=\sinh \left(L s_{0}\right), \quad \text { or } \quad \sinh \left(L s_{0}\right)=\left(L s_{0}\right) \sqrt{\frac{2}{p L^{2}}} .
$$

This equation is of the form $\sinh x=\eta x$, with $x>0$. Denoting the solution as $x(\eta)$, one finds (using the implicit function theorem) that $x^{\prime}(\eta) \sim \eta^{-1}$ as $\eta \rightarrow+\infty$, hence $x(\eta) \sim \ln \eta$. In our case, we thus have:

$$
a<s_{0} \sim-\frac{1}{2 L} \ln p \quad \text { as } p \rightarrow 0 .
$$

Mass. This is the integral of both sides of (B.1).

$$
\begin{aligned}
M & :=\int_{0}^{L} p \mathrm{e}^{u(x)} \mathrm{d} x=2 a[\operatorname{coth}(b-a L)-\operatorname{coth} b] \\
& =\sqrt{2 p \mathrm{e}^{m}+4 a^{2}}-\sqrt{2 p+4 a^{2}} .
\end{aligned}
$$

The main result of this appendix is the following.

Proposition Appendix B.1. If $M$ remains bounded under (B.3), then the parameters $p, m$ satisfy:

$$
p \mathrm{e}^{\beta m} \rightarrow 0 \text { for all } \beta<1 \text {. }
$$

Proof. We begin by proving (by contradiction) that, if $M$ remains bounded:

$$
p \mathrm{e}^{m} / a^{2} \rightarrow 0 \quad \text { under (B.3). }
$$


Assume that this condition does not hold. Then there exist sequences $p_{n} \rightarrow 0, m_{n} \rightarrow$ $+\infty$, such that the corresponding values of $a$ eventually satisfy $p_{n} \mathrm{e}^{m_{n}} / a_{n}^{2} \geq C>0$. The values of the mass are then bounded from below as:

$$
M_{n} \geq a_{n}[\sqrt{C+4}-\sqrt{4+o(1)}] \rightarrow+\infty,
$$

and we get the contradiction. Combining (B.10) and (B.7), we get:

$$
p \mathrm{e}^{m} / \ln ^{2} p \stackrel{(\mathrm{B} .3)}{\longrightarrow} 0, \quad \text { hence } \quad p^{1+\nu} \mathrm{e}^{m} \rightarrow 0 \quad \text { and } \quad p \mathrm{e}^{\beta m} \rightarrow 0,
$$

for any $\nu>0$ and $\beta=1 /(1+\nu)<1$.

Extension to a subsolution on a larger interval. We already used in (B.8) the fact that $u^{\prime}(L)=-2 a \operatorname{coth} b$, which goes to $-\infty$ under (B.3). Therefore, defining the function $\widetilde{u}$ as

$$
\widetilde{u}(x):=u(x) \text { for } x \leq L, \quad \widetilde{u}(x):=-(2 a \operatorname{coth} b)(x-L)+\frac{p}{2}(x-L)^{2} \text { for } x \geq L,
$$

we see that $\widetilde{u} \in C^{1}\left(\mathbb{R}^{+}\right)$. For $x>L$, there holds $\widetilde{u}^{\prime \prime}(x)=p \geq p \mathrm{e}^{\widetilde{u}(x)}$ as long as $\widetilde{u}(x) \leq 0$. Thus, if $\widetilde{u}\left(L^{\prime}\right) \leq 0$ for some $L^{\prime}>L-$ which is eventually true if $L^{\prime}$ is fixed - then $\widetilde{u}$ is a subsolution to (B.1)-(B.2) on the interval $\left(0, L^{\prime}\right)$.

\section{Appendix C. Details on the singular complement method}

This method is described in Ref. 7. In this section, we recall the practical numerical details of the method, and we give the result of a straightforward but tedious calculation involved in it. We assume the reentrant corner located at $(0,0)$ with angle $\pi / \alpha, \alpha \in(1 / 2,1)$ and its two edges at angles $\theta=0$ and $\pi / \alpha$. We denote $\Gamma_{s} \subset \Gamma_{1}$ the union of these two edges.

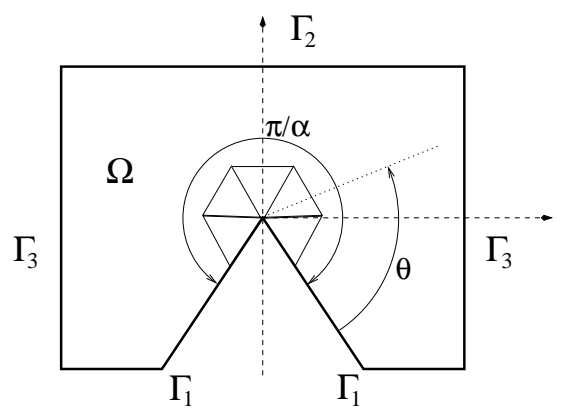

Fig. 8. The domain used in the numerical tests of $\S 6$. 


\section{C.1. Computing the dual singularity $P_{s}$}

This is done by splitting this function into an analytically known principal part $P_{p}$ and a smoother remainder $\tilde{P}$, as in (4.8)-(4.9). The remainder $\tilde{P} \in H^{1}(\Omega)$ will be approximated by $\tilde{P}^{h} \in V^{h}$, and so we define $P_{s}^{h}:=P_{p}+\tilde{P}^{h}$. Moreover we define the approximation $\varkappa^{h}$ of $\varkappa=\left\|P_{s}\right\|_{L^{2}}^{2}$ by:

$$
\begin{aligned}
\varkappa^{h}=\left(P_{s}^{h}, P_{s}^{h}\right) & =\left\|P_{p}\right\|_{L^{2}}^{2}+2\left(P_{p}, \tilde{P}^{h}\right)+\left(\tilde{P}^{h}, \tilde{P}^{h}\right) \\
& =\left\|P_{p}\right\|_{L^{2}}^{2}+2 \sum_{i}\left(P_{p}, \varphi_{i}\right)\left\{\tilde{P}^{h}\right\}_{i}+\left\{\tilde{P}^{h}\right\}^{\top} \mathbb{M}\left\{\tilde{P}^{h}\right\}
\end{aligned}
$$

Some care must be taken in computing $\left\|P_{p}\right\|_{L^{2}}^{2}$ and $\left(P_{p}, \varphi_{i}\right)$ see $\S$ C.3.

\section{C.2. Computing the primal singularity $\phi_{s}$}

In a similar way, an approximation of the primal singularity function $\phi_{s}=\Phi\left[P_{s}\right]$ is obtained by splitting it into a principal part $\phi_{p}=\varkappa r^{\alpha} \sin (\alpha \theta)$ and a remainder $\tilde{\phi} \in H^{2}(\Omega)$, cf. (4.3):

$$
\phi_{s}=\varkappa r^{\alpha} \sin (\alpha \theta)+\tilde{\phi}
$$

with $\tilde{\phi}$ solution to:

$$
-\Delta \tilde{\phi}=P_{s} \text { in } \Omega, \quad \tilde{\phi}=-\phi_{p} \text { on } \Gamma_{1} \cup \Gamma_{2}, \quad \partial_{\nu} \tilde{\phi}=-\partial_{\nu} \phi_{p} \text { on } \Gamma_{3} .
$$

In numerical computations, we use $P_{s}^{h}=P_{p}+\tilde{P}^{h}$ as right-hand side instead of $P_{s}$, and $-\varkappa^{h} \Pi_{I}^{h}\left(r^{\alpha} \sin (\alpha \theta)\right),-\varkappa^{h} \Pi_{I}^{h} \partial_{\nu}\left(r^{\alpha} \sin (\alpha \theta)\right)$ as boundary data. Thus we get a finite element solution $\tilde{\phi}^{h}$. To obtain an approximation of $\phi_{s}$ in $V^{h}$ we define:

$$
\phi_{s}^{h}=\tilde{\phi}^{h}+\varkappa^{h} \Pi_{I}^{h}\left(r^{\alpha} \sin (\alpha \theta)\right) .
$$

Here the difficulty is to compute the right-hand side of the finite element equations, that is, the scalar products:

$$
\left(P_{s}^{h}, \varphi_{i}\right)=\left(P_{p}, \varphi_{i}\right)+\left(\tilde{P}^{h}, \varphi_{i}\right)=\left(P_{p}, \varphi_{i}\right)+\mathbb{M}_{i}\left\{\tilde{P}^{h}\right\},
$$

where $\mathbb{M}_{i}$ denotes the $i$-th row of the mass matrix. So, again, the difficulty is reduced to computing the $\left(P_{p}, \varphi_{i}\right)$ terms.

\section{C.3. Details on computing $\left(P_{p}, \varphi_{i}\right)$ and $\left\|P_{p}\right\|_{L^{2}}^{2}$}

Computing $\left(P_{p}, \varphi_{i}\right)$ terms

If the first layer of triangles around the reentrant corner is made of isosceles triangles (see Figure 8), an exact calculation of the non-vanishing parts of the terms $\left(P_{p}, \varphi_{i}\right)$ can be done when $i$ is the index of a vertex on the first layer. Indeed, after tedious calculations one can show the following result: if $v$ is an affine function defined on the isosceles triangle $T$ with vertex $\boldsymbol{P}_{0}=(0,0)$ : 


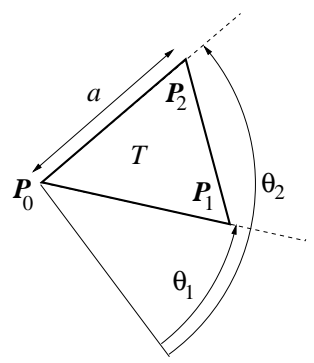

then $I_{T}:=\int_{T} P_{p}(x, y) v(x, y) \mathrm{d} x \mathrm{~d} y$ is given by:

$$
\begin{aligned}
I_{T}=\frac{2 a^{2-\alpha} \cos \varpi}{1-\alpha} & {\left[C \sin \left(\alpha \theta_{m}\right) \sin ((1-\alpha) \varpi)\right.} \\
+D & \left.\frac{\cos \left(\alpha \theta_{m}\right)}{(2-\alpha) \sin \varpi}(\sin (\alpha \varpi)-\alpha \sin \varpi \cos ((1-\alpha) \varpi))\right]
\end{aligned}
$$

where we have set:

$$
\begin{aligned}
& \varpi=\left(\theta_{2}-\theta_{1}\right) / 2, \quad \theta_{m}=\left(\theta_{1}+\theta_{2}\right) / 2 ; \\
& C=\frac{1}{\pi} \frac{1}{(3-\alpha)}\left(\frac{v_{0}}{2-\alpha}+\frac{v_{1}+v_{2}}{2}\right), \quad D=\frac{1}{\pi} \frac{1}{(3-\alpha)}\left(\frac{v_{2}-v_{1}}{2}\right) ; \\
& v_{i}=v\left(\boldsymbol{P}_{i}\right), \quad \text { for } i=0,1,2 .
\end{aligned}
$$

On the other triangles the function $P_{p}$ is smooth, so we use a numerical quadrature formula with 7 interior points (Radon's rule, p. 314 in Ref. 22). The requirement to have a layer of isosceles triangles around the corner is easily feasible when using the versatile (and very fast) meshing code triangle. ${ }^{21}$

\section{Computing $\left\|P_{p}\right\|_{L^{2}}^{2}$}

Our approach consists in using an exact integration in $r$ which "kills" the singularity, followed by a numerical integration in $\theta$. This can be done for any sub-domain $\omega \subset \Omega$ star-shaped around the reentrant corner, with an external border known as a function of $\theta$, say, $\omega=[r<R(\theta)]$. We obviously get:

$$
\left\|P_{p}\right\|_{L^{2}(\omega)}=\frac{1}{(2-2 \alpha) \pi^{2}} \int_{0}^{\frac{\pi}{\alpha}} R(\theta)^{2-2 \alpha} \sin ^{2}(\alpha \theta) \mathrm{d} \theta .
$$

In the case of Figure 8, we can take $\omega$ as the whole domain $\Omega$. Using the symmetry of the domain, the resulting integral is split into three parts (bottom (right) border, right border and half right top border) corresponding to smooth expressions of the function $R(\theta)$, which are computed with the well-known quadpack ${ }^{20}$ routine dqag.

\section{Acknowledgments}

F.K. wishes to thank the INRIA project-team CALVI for its financial support during his post-doctorate in Nancy, when part of this work was done. 


\section{References}

1. J.P. Aubin and I. Ekeland, Applied Nonlinear Analysis (John Wiley \& Sons, 1984).

2. N. Ben Abdallah and J. Dolbeault, Relative entropies for kinetic equations in bounded domain (irreversibility, stationary solution, uniqueness), Arch. Rational Mech. Anal. 168 (2003) 253-298.

3. L. Bieberbach, $\Delta u=\mathrm{e}^{u}$ und die automorphen Funktionen, Math. Ann. 77 (1916) $173-212$.

4. M. Bostan, Weak solutions for the Vlasov-Poisson initial-boundary value problem with bounded electric field, Chin. Ann. Math. Ser. B 28 (2007) 389-420.

5. M.J. Cáceres, J.A. Carrillo and J. Dolbeault, Nonlinear stability in $L^{p}$ for a confined system of charged particles, SIAM J. Math. Anal. 34 (2002) 478-494.

6. F.F. Chen, Introduction to Plasma Physics and Controlled Fusion (Plenum Press 1984).

7. P. Ciarlet, Jr. and J. He, The singular complement method for 2D scalar problems, C. R. Math. Acad. Sci. Paris 336 (2003) 353-358.

8. J. Dolbeault, Free energy and solutions of the Vlasov-Poisson-Fokker-Planck system: External potential and confinement (large time behavior and steady states), J. Math. Pures Appl. (9) 78 (1999) 121-157.

9. J. García Melián, J.D. Rossi and J. Sabina, Existence, asymptotic behavior and uniqueness for large solutions to $\Delta u=\mathrm{e}^{q(x) u}$, Advanced Nonlinear Studies 9 (2009) 395-424.

10. J.C. Gilbert and C. Lemaréchal, Some numerical experiments with variable-storage quasi-Newton algorithms, Math. Programming 45 (Ser. B) (1989) 407-435.

11. P. Grisvard, Elliptic problems in nonsmooth domains, Monographs and Studies in Mathematics 24 (Pitman, Boston, 1985).

12. P. Grisvard, Singularities in Boundary Value Problems (Masson, Paris, 1992).

13. Y. Guo, Global weak solutions of the Vlasov-Maxwell system with boundary conditions, Commun. Math. Phys. 174 (1993) 245-263.

14. Y. Guo, Singular Solutions of the Vlasov-Maxwell System on a Half Line, Arch. Rational Mech. Anal. 131 (1995) 241-304.

15. H.J. Hwang, Regularity for the Vlasov-Poisson system in a convex domain, SIAM J. Math. Anal. 36 (2004) 121-171.

16. H.J Hwang and J.J.L. Velázquez, Global existence for the Vlasov-Poisson system in bounded domains, Arch. Ration. Mech. Anal. 195 (2010) 763-796.

17. S. Labrunie, J.A. Carrillo and P. Bertrand, Numerical simulation on hydrodynamic and quasi-neutral approximations for collisionless two-species plasmas, J. Comput. Phys. 200 (2004) 267-298.

18. A.C. Lazer and P.J. McKenna, On a problem of Bieberbach and Rademacher, Nonlinear Anal. 21 (1993) 327-335.

19. J.L. Lions, Quelques méthodes de résolution des problèmes aux limites non linéaires (Dunod, Paris, 1969).

20. R. Piessens, E. De Doncker-Kapenga and C.W. Überhuber, QUADPACK: a subroutine package for automatic integration (Springer, 1983).

21. J.R. Shewchuk, Triangle: Engineering a 2D Quality Mesh Generator and Delaunay Triangulator, in Applied Computational Geometry: Towards Geometric Engineering (editors: Ming C. Lin and Dinesh Manocha), Lecture Notes in Computer Science, 1148, pp. 203-222 (Springer, 1996).

22. A.H. Stroud, Approximate calculation of multiple integrals (Prentice-Hall, Englewood Cliffs, 1971). 